\title{
Generalized Modular Transformations in $(3+1)$ D Topologically Ordered Phases and Triple Linking Invariant of Loop Braiding
}

\author{
Shenghan Jiang, Andrej Mesaros, and Ying Ran \\ Department of Physics, Boston College, Chestnut Hill, Massachusetts 02467, USA
}

(Received 7 April 2014; published 10 September 2014)

\begin{abstract}
In topologically ordered quantum states of matter in $(2+1) \mathrm{D}$ (spacetime dimensions), the braiding statistics of anyonic quasiparticle excitations is a fundamental characterizing property that is directly related to global transformations of the ground-state wave functions on a torus (the modular transformations). On the other hand, there are theoretical descriptions of various topologically ordered states in $(3+1) \mathrm{D}$, which exhibit both pointlike and looplike excitations, but systematic understanding of the fundamental physical distinctions between phases, and how these distinctions are connected to quantum statistics of excitations, is still lacking. One main result of this work is that the three-dimensional generalization of modular transformations, when applied to topologically ordered ground states, is directly related to a certain braiding process of looplike excitations. This specific braiding surprisingly involves three loops simultaneously, and can distinguish different topologically ordered states. Our second main result is the identification of the three-loop braiding as a process in which the worldsheets of the three loops have a nontrivial triple linking number, which is a topological invariant characterizing closed two-dimensional surfaces in four dimensions. In this work, we consider realizations of topological order in $(3+1) \mathrm{D}$ using cohomological gauge theory in which the loops have Abelian statistics and explicitly demonstrate our results on examples with $Z_{2} \times Z_{2}$ topological order.
\end{abstract}

DOI: 10.1103/PhysRevX.4.031048

\section{INTRODUCTION}

Topologically ordered quantum phases of matter in $(2+1) \mathrm{D}$ have been intriguing since their discovery decades ago (see Ref. [1] and references therein), due to exotic properties such as fractionalized quasiparticles with anyonic quantum braiding statistics [2,3]. Early on, it was realized that in such phases the topological degeneracy of the ground state on the torus corresponds to the number of types of particle excitations (superselection sectors) [4]. Furthermore, it was shown that the matrix of Berry's phases experienced by the ground states under the modular transformations of the torus, the $S$ and $T$ transformations [Fig. 1(a)], are directly related to the quantum statistics of the quasiparticles [2]. In fact, to date the most fundamental conjecture remains that the matrices of $S, T$ contain complete information about a topological order [2]. Therefore, one can view the modular $S, T$ matrices as the "nonlocal order parameters" in a topologically ordered phase [5].

However, in three spatial dimensions, some fundamental questions are yet completely unresolved: Is there a physical

Published by the American Physical Society under the terms of the Creative Commons Attribution 3.0 License. Further distribution of this work must maintain attribution to the author(s) and the published article's title, journal citation, and DOI.
Subject Areas: Condensed Matter Physics, Strongly Correlated Materials way to characterize different topological orders in $(3+1)$ D? Can braiding of excitations help us in the characterization? Clearly the problem is much more complex, since in 3D there are generically both pointlike and looplike excitations, and their geometric interplay is rich. If some type of braiding can help us characterize the topological order in $(3+1) \mathrm{D}$, what is the topological property of that braiding process that is relevant?

Motivated by the fundamental role of modular transformations of the torus in $(2+1) \mathrm{D}$ systems, our approach to these questions is based on considering the analogous transformations on the three-torus (e.g., a cube with

(a)
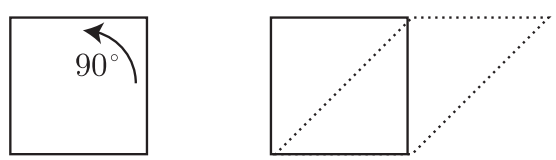

(b)

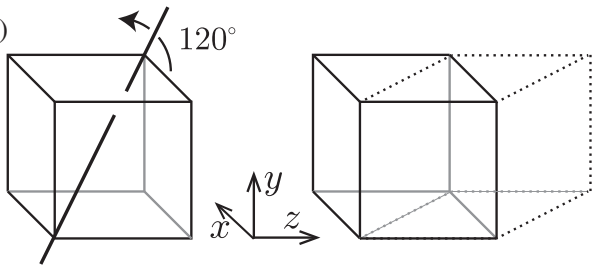

FIG. 1. $S$ (left) and $T$ (right) transformations on the (a) twotorus and (b) three-torus, which are defined by periodic boundary conditions. 
periodic boundary conditions). The modular transformations $S, T$ on the torus generate the group $S L(2, Z)$, which represents the different classes of continuous transformations on the torus [6]. In $(3+1) \mathrm{D}$ quantum states, the analogue is the three-torus, which also has just two associated transformations $S, T$, generators of the $S L(3, Z)$ group [7,8], namely, a $120^{\circ}$ rotation through a diagonal of the periodic cube and a shear, respectively [Fig. 1(b)]. Very recently, it has been conjectured that exactly these kinds of transformations can be used to characterize topological order in any dimension [8].

One way to study topologically ordered states is by using the exactly solvable models of discrete gauge theories introduced by Dijkgraaf and Witten $[9,10]$. Although these theories in $(2+1) \mathrm{D}$ do not provide an exhaustive classification of all possible topological orders [11], they describe a physically interesting set of states. Most importantly for this work, such cohomological gauge theories with gauge group $G$ are naturally defined in any spatial dimension, allowing us to study $(3+1) \mathrm{D}$ topological orders. They also host both pointlike and looplike excitations, namely, gauge charges and flux loops, respectively. For simplicity, we restrict our study to the case of Abelian groups $G$, and then additionally to cases where loops have only Abelian braiding.

Our approach to the $(3+1) \mathrm{D}$ problem is based on generalizing some aspects of the $(2+1) \mathrm{D}$ case, which we review here. It is well known that topological operators, which describe tunneling of quasiparticle across the periodic 2D system, obey a nontrivial algebra. A certain product of these operators gives the identity operator times a complex number, which equals an $\mathcal{S}$-matrix element, and also equals the quasiparticle statistics $[3,12]$. To generalize to three dimensions, we need the topological content of this relation, which is revealed using a picture. Figure 2(c) depicts the expectation value of the relevant product of tunneling operators as a time sequence of events where particle-antiparticle pairs tunnel across the periodic system. Note that the initial and final state in the picture are the same. The four worldline segments in this process, belonging to two quasiparticle types, can be connected to reveal two linked worldlines, Fig. 2(d). The picture shows how the $\mathcal{S}$-matrix element and the braiding statistics can be seen in the linking of worldlines. One can detect the linking of worldlines in a purely algebraic way, without drawing the two figures, by calculating the linking topological invariant of the two worldlines. This invariant simply counts the number of links in the one-dimensional worldlines living in the three-dimensional spacetime.

There is another way to relate an $\mathcal{S}$-matrix element to a topological property of the quasiparticle braiding process in $(2+1) \mathrm{D}$, which will be important for our generalization to $(3+1)$ D. Recently, the connection between modular transformations on the ground-state manifold in $(2+1) \mathrm{D}$ and the statistics of quasiparticles was further exposed by the (a)
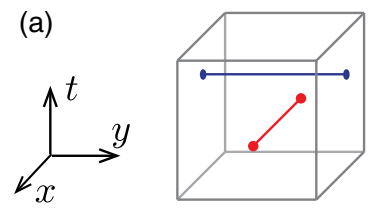

(c)
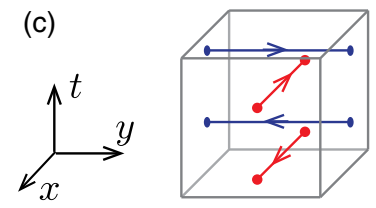

(d)
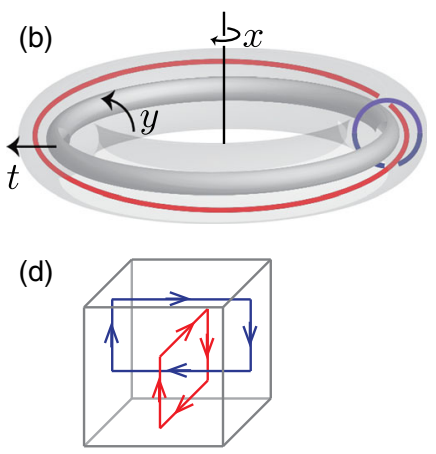

FIG. 2. An $\mathcal{S}$-matrix element and braiding in $(2+1) \mathrm{D}$. (a) Matrix element equals an overlap of two MESs, shown as a time sequence. Any MES is defined by action of particle tunneling operator along $x$ on the appropriate reference state defined by $x$ direction. The MES at later time (blue) has been acted on by $S$ modular transformation, so the tunneling is along $y$, and the final reference state is defined by $y$ direction. (b) Embedding the spacetime process of (a) in three dimensions shows that the two worldlines are linked. Time grows in the radial direction as shown, so the spacetime of (a) spans the volume of a toroidal slab. (c) Alternatively, the matrix element equals a product of tunneling operators, also presented as a time sequence. Arrows mark the action of tunneling operator and its inverse. Because of taking the expectation value of this operator product, the initial and final state are the same, in contrast to (a). (d) Connecting the worldlines from (c), one obtains two worldlines that are linked.

introduction of minimum entropy states (MES), a special choice of basis in the ground-state manifold [13]. Namely, an $\mathcal{S}$-matrix element is related to an overlap of two MESs [13]. This relation is due to the fact that every MES can be created by the action of a quasiparticle tunneling operator on the appropriate reference state [13]. A pictorial representation again uncovers the topological content in this understanding: Figure 2(a) depicts the overlap of two MESs as a time sequence of applying quasiparticle tunneling operators. Note that here the initial and final states in the picture are different reference states, being rotated by $90^{\circ}$. In this case, we immediately obtain the two worldlines, since each MES in the overlap contributes one. The picture reveals that the two worldlines in this process are linked when the $(2+1) \mathrm{D}$ spacetime process is embedded in three-dimensional space as in Fig. 2(b). This approach again connects the measurement of braiding statistics $(\mathcal{S}$-matrix element) in $(2+1) \mathrm{D}$ to the linking of particle worldlines.

In this paper, we generalize both of the above approaches to a $(3+1) \mathrm{D}$ cohomological gauge theory and obtain some surprising relations between the matrix elements of the three-torus $S, T$ transformations and the braiding of excitations. Noticing that the line, which represents a quasiparticle tunneling operator across a periodic direction on the two-torus, becomes a membrane, which represents the tunneling of a flux loop across two periodic directions in the three-torus, we construct the appropriate membrane 
operators as well as the MES on the three-torus. We show that in $(3+1) \mathrm{D}$ topological order, the nontrivial matrix elements of the $S, T$ transformations in the MES basis are due to a nontrivial algebra of the topological membrane operators, analogously to the $(2+1) \mathrm{D}$ case.

Next, we generalize to $(3+1) \mathrm{D}$ the two $(2+1) \mathrm{D}$ approaches described above. Namely, we study the spacetime process representing the nontrivial product of membrane operators, i.e., the loop tunneling operators, as well as the spacetime process of overlapping two MESs. Both calculations give the same value, which equals a matrix element of the three-torus $S$ transformation. This value also represents a topological quantum phase accrued during some loop spacetime process, and the main question becomes, What is the nature of this loop process? In the analogous situation in $(2+1) \mathrm{D}$, we describe that the particle process is simple braiding, which is characterized by the linking of worldlines.

Most strikingly, we argue that the obtained $\mathcal{S}$-matrix elements in $(3+1) \mathrm{D}$ relate to certain braiding processes involving three loops simultaneously. This is surprising since there is a simple, seemingly fundamental, braiding process of just two loops, where one loop traces out a torus enclosing the other loop, which is relevant in other physical contexts $[14,15]$.

To uncover the topological underpinning of the two studied $(3+1) \mathrm{D}$ spacetime processes, we consider the topological invariants of the loop worldsheets in both of them. Although we cannot draw the pictures of twodimensional worldsheets living in the four-dimensional spacetime, in analogy with Fig. 2, we find through calculation that the loop worldsheets in both spacetime processes are described by the exact same nontrivial values of the triple linking number (TLN) [16]. The triple linking number is an integer invariant of closed surfaces in four dimensions, and can be seen as the $(3+1) \mathrm{D}$ generalization of the linking number of closed lines in three dimensions, which is relevant for particles in the $(2+1) \mathrm{D}$ case.

The TLN is obviously the fundamental, truly threedimensional descriptor of the relevant three-loop braiding process, which is revealed in the context of this paper. To capture its meaning in a tangible way, in this paper we also construct a time sequence of a process involving three loops, shown in Fig. 3, such that the three loop worldsheets in spacetime exhibit exactly the same values of TLN as obtained in the two processes above. This time sequence shows the physical braiding of three flux loops resulting in a topological quantum phase that measures the properties of the underlying $(3+1) \mathrm{D}$ topological order (at least for cohomological gauge theory), and equals a matrix element of the three-dimensional $S$ modular transformation.

This paper is organized as follows. In Sec. II, we define the exactly solvable models in $(3+1) \mathrm{D}$, which are classified by cohomology group and can be viewed as an

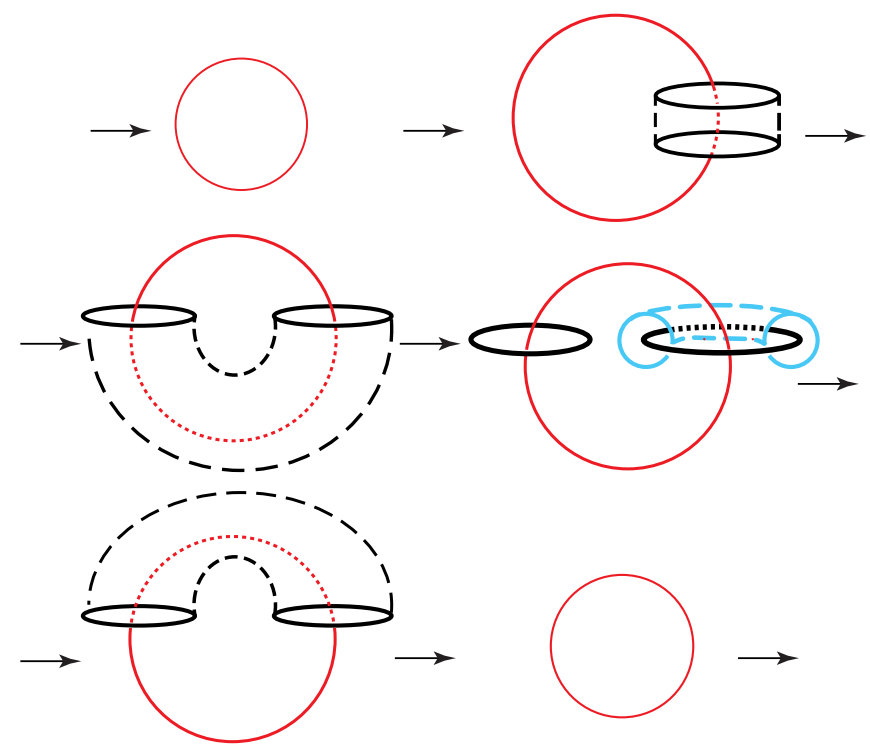

FIG. 3. Time sequence for three-flux-loop braiding. This process has nontrivial triple linking number of three worldsheets. First, loop $G$ (red) is created and grows, forming the $G$ worldsheet. Then, loop $H$ (black) emerges, encircling loop $G$ halfway. Then, loop $F$ (blue) completely encircles loop $H$. After this, loop $H$ finishes the route around loop $G$. Finally, loop $G$ is annihilated.

extension of the Dijkgraaf-Witten theory to $(3+1)$ D. In Sec. III, we put these models on three-torus and find the ground state manifolds. Particularly, we find a MES basis, which is useful for interpretation. Further, we construct membrane operators, defined as operators mapping between MESs. We work out the modular transformations on the MES basis in Sec. IV. We find modular transformations to be directly related to braiding statistics of flux loops and particles. We show this by both geometric and algebraic methods. In Sec. V, we solve these models for some illuminating examples.

\section{COHOMOLOGICAL GAUGE THEORY IN $(\mathbf{3}+\mathbf{1})$ D}

In this section, we define the cohomological gauge theory for a general manifold in $(3+1) \mathrm{D}$, based on the Dijkgraaf-Witten topological invariant. The theory is topological and defined by a discrete gauge group $G$. However, there are distinct topologically ordered states for a fixed $G$, and in $(3+1) \mathrm{D}$ they are classified by the fourth cohomology group of $G$ with coefficients in $U(1)$, namely, $H^{4}(G, U(1))$. In Appendix A, we give a brief review of cohomology concepts relevant for the rest of the paper, while referring the reader to Refs. $[17,18]$ for more details.

In this paper, we work in $(3+1) \mathrm{D}$, and therefore, the theory is defined using the 4-cocycle (sometimes we call it simply cocycle) $\omega$, for which the cocycle condition becomes 


$$
\begin{aligned}
& \omega\left(g_{2}, g_{3}, g_{4}, g_{5}\right) \cdot \omega\left(g_{1}, g_{2} \cdot g_{3}, g_{4}, g_{5}\right) \cdot \omega\left(g_{1}, g_{2}, g_{3}, g_{4} \cdot g_{5}\right) \\
& \quad=\omega\left(g_{1} \cdot g_{2}, g_{3}, g_{4}, g_{5}\right) \cdot \omega\left(g_{1}, g_{2}, g_{3} \cdot g_{4}, g_{5}\right) \cdot \omega\left(g_{1}, g_{2}, g_{3}, g_{4}\right),
\end{aligned}
$$

where $\omega \in H^{4}(G, U(1))$, and $g_{i} \in G$. In this paper, we use the "canonical" 4-cocycle, meaning that $\omega\left(g_{1}, g_{2}\right.$, $\left.g_{3}, g_{4}\right)=1$ if any of $g_{1}, g_{2}, g_{3}, g_{4}$ is equal to 1 (the identity element of group $G$ ).

The gauge theory is now defined by using $\omega$ to construct topological invariants of a 4D manifold. For a given 4D manifold $M$ without boundary, one can triangulate it using a finite number of 4-simplices $[19,20]$. The 4-simplex is a higher-dimensional analogue of a regular polyhedron and can be constructed from a tetrahedron by adding a fifth vertex and moving it into the fourth dimension so that all the edges from it to the four original vertices are the same length as the tetrahedron's edges. The triangulation basically requires completely filling the manifold $M$ with 4-simplices without overlaps. The vertices of this triangulation are then ordered arbitrarily, and the ordering is represented by assigning arrows going from the lower to the higher-ordered vertex on each edge, Fig. 4. Let us denote a 4-simplex of the triangulation, together with the ordering of its vertices, by $\sigma_{I}$, where $I=1,2, \ldots, S$ labels 4 -simplices and $S$ is the total number of 4-simplices in $M$. Next, one defines a coloring $\varphi$ of all the edges in the triangulation by assigning a group element to them. Let us denote the group element assigned to the bond connecting vertices $j$ and $i$ as $g_{i j}$, following the ordering from $j$ to $i$ : $j \rightarrow i$; we then automatically assign $g_{j i}=g_{i j}^{-1}$. In addition, the three assigned group elements for any given face must satisfy the constraint $g_{i j} \cdot g_{j k} \cdot g_{k i}=\mathbf{1}$, and $i, j, k$ are the three vertices of the face. This constraint is the "zero-flux rule."

With these definitions, one can assign a $U(1)$ phase to every 4-simplex by computing $\omega^{\varepsilon}\left(g_{54}, g_{43}, g_{32}, g_{21}\right)$, where $\varepsilon=\operatorname{sgn}[\operatorname{det}(\overrightarrow{12}, \overrightarrow{23}, \overrightarrow{34}, \overrightarrow{45})]$ determines the chirality of the simplex, as shown in Fig. 4 [21]. For a given coloring $\varphi$

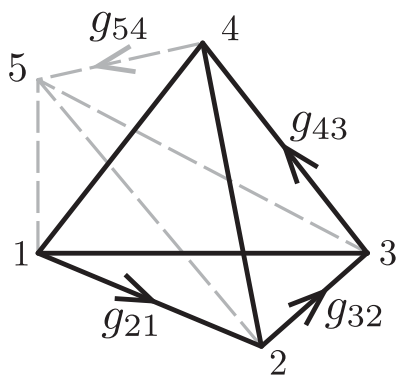

FIG. 4. The 4-cocycle $\omega$ assigns a $U(1)$ complex number $\omega^{\varepsilon}\left(g_{54}, g_{43}, g_{32}, g_{21}\right)$ to a 4-simplex, where $\varepsilon$ is the chirality of the 4-simplex, defined as $\varepsilon=\operatorname{sgn}[\operatorname{det}(\overrightarrow{12}, \overrightarrow{23}, \overrightarrow{34}, \overrightarrow{45})]$. The dashed lines represent that the vertex 5 has a different coordinate in the fourth dimension (time) with respect to the other vertices. and simplex $\sigma_{I}$, we label this $U(1)$ phase as $W\left(\sigma_{I}, \varphi\right)^{\varepsilon\left(\sigma_{I}\right)}$. Finally, one can compute the product of all $W$ for the simplices: $\prod_{I=1}^{S} W\left(\sigma_{I}, \varphi\right)^{\epsilon\left(\sigma_{I}\right)}$. For a given coloring $\varphi$, we have one such product. The key result [9] is that the complex number

$$
Z_{M}=\frac{1}{|G|^{V}} \sum_{\substack{\rho \in \text { all } \\ \text { possible } \\ \text { colorings }}} \prod_{I=1}^{S} W\left(\sigma_{I}, \varphi\right)^{\epsilon\left(\sigma_{I}\right)}
$$

where $|G|$ is the number of elements in group $G$ and $V$ is the number of vertices in the triangulation, is a topological invariant of the manifold $M$. More precisely, $Z_{M}$ does not depend on the triangulation and the ordering of vertices (while different colorings are already summed over), owing to the cocycle condition in Eq. (1). One can further show that equivalent cocycles (i.e., cocycles differing by a coboundary) give the same value of $Z_{M}$ [9].

The topological invariant $Z_{M}$ is exactly the partition function of the cohomological gauge theory, which is a topological quantum field theory for discrete gauge group $G$ in $(3+1)$ D. It is the higher-dimensional version of the Dijkgraaf-Witten theory [9,22], and it depends only on inequivalent elements in $H^{4}(G, U(1))$.

\section{A. Exactly solvable models}

We define our exactly solvable models in $(3+1) \mathrm{D}$ as Hamiltonian versions of the cohomological gauge theory. We consider space triangulated using a tetrahedron lattice with oriented edges (bonds), where these orientations are compatible with some ordering of lattice sites, and assign an element $g_{i j} \in G$ to each oriented edge $j \rightarrow i$, according to the above discussion.

An arbitrary quantum state in the Hilbert space $\mathcal{H}$ of our model is then labeled by $|a\rangle=\left|\left\{g_{i j}\right\}\right\rangle$. The building block for the Hamiltonian is the operator $\hat{B}_{p}^{s}$ labeled by a group element $s \in G$ and a "plaquette" $p$ containing all 3 -simplices (tetrahedra) that share the vertex $i$. The plaquette operator acts on group elements on the edges that share $i$. To define its action, we introduce an additional edge rising into the fourth dimension, connecting $i$ to an auxiliary vertex $i^{\prime}$. To edge $i \rightarrow i^{\prime}$, we assign the element $s \in G$. The group elements are changed as

$$
\begin{aligned}
g_{i j} & \rightarrow s \cdot g_{i j}, \\
g_{k i} & \rightarrow g_{k i} \cdot s^{-1},
\end{aligned}
$$

and these new values are represented on auxiliary edges $i^{\prime} \rightarrow j$ and $k \rightarrow i^{\prime}$. Further, the nonzero matrix elements of $\hat{B}_{p}^{s}$, namely, $B_{p}^{s}=\left\langle f(s)\left|\hat{B}_{p}^{s}\right| i\right\rangle$, are assigned the following quantum amplitude: 


$$
B_{p}^{s} \equiv \prod_{I=1}^{6} W\left(\sigma_{I}, \varphi\right)^{\varepsilon\left(\sigma_{I}\right)}
$$

where the 4-simplices $\sigma_{I}$ are built by triangulating the 4D volume formed by the tetrahedra in the plaquette $p$ and the auxiliary edges.

It is important to note that the zero-flux rule is by construction satisfied on all faces (triangles) of 4-simplices, if it is satisfied in the tetrahedra of $p$, and this must be imposed for the $B_{p}^{s}$ to be well defined. We can then define the plaquette operators $\hat{B}_{p}$ as having matrix elements

$$
B_{p}=\frac{1}{|G|} \sum_{s \in G} B_{p}^{s}
$$

The $\hat{B}_{p}$ are projectors, which can be easily checked using the cocycle property to show $\left\langle f\left|\hat{B}_{p}^{s} \hat{B}_{p}^{s^{\prime}}\right| i\right\rangle=B_{p}^{s \cdot s^{\prime}}$, which then implies $\left\langle f\left|\hat{B}_{p} \hat{B}_{p}\right| i\right\rangle=B_{p}$. Similarly, it can be shown that the plaquette operators commute, $\left[B_{p}, B_{p^{\prime}}\right]=0$, $\forall p, p^{\prime}$.

Let us also introduce the operator $Q_{t}$, which projects flux in a triangle $t$ to zero; i.e., it enforces the zero-flux rule. Then, the Hamiltonian takes the form

$$
H=-\sum_{t} Q_{t}-\sum_{p} \hat{B}_{p} \prod_{t \in p} Q_{t},
$$

where the label $t \in p$ enumerates all the triangles making up the plaquette $p$. As mentioned above, the factor $\prod_{t \in p} Q_{t}$ is actually crucial to ensure that $H$ is well defined. Further, it is easy to see that the plaquette operator term $\hat{B}_{p} \prod_{t \in p} Q_{t}$ actually commutes with the projectors $Q_{t^{\prime}}$. Since all the terms in $H$ commute with each other, the model is exactly solvable.

Let us briefly mention the connection of the Hamiltonian formulation to the gauge theory, which is exhibited in the ground-state manifold. Since all the terms in $H$ are projectors, the ground-state manifold is the image of the projector $P=\prod_{p} \hat{B}_{p} \prod_{t \in p} Q_{t}$. On the other hand, $P$ is exactly the projector defining the cohomological gauge theory on the 4D manifold having two copies of our spatial manifold $M$ as boundaries (see Ref. [18] for details). The ground-state sector of $H$, to which $P$ projects with eigenvalue 1 , is also the groundstate sector of the cohomological gauge theory [9] defined on $M$.

\section{B. Geometrical reduction of 4-cocycles}

In this section, we present some cohomology equations for reducing the 4-cocycle to lower-order cocycles, and explain their geometric meaning. These equations crucially simplify all following calculations. From now on, we focus on Abelian groups $G$ for convenience.

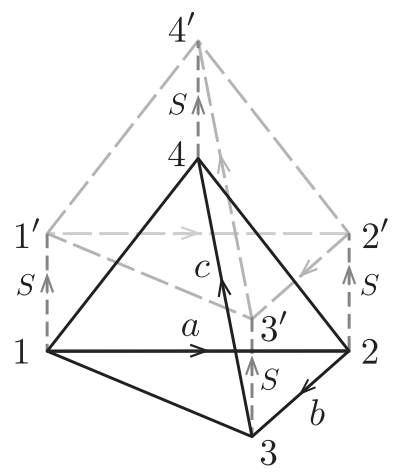

FIG. 5. Geometric meaning of 3-cocycle $\beta_{s}(c, b, a)$ corresponds to evolution (along fourth dimension) of tetrahedron [1234] to $\left[1^{\prime} 2^{\prime} 3^{\prime} 4^{\prime}\right]$.

First, we consider a triangulated 4D manifold in Fig. 5, with the shown coloring. (Note that some edges needed for full 4D triangulation are omitted, but coloring and ordering are fully defined.) The $U(1)$ phase calculated from all the 4 -simplices spanning this $4 \mathrm{D}$ volume, with the 4-cocycle $\omega$ given, equals $\beta_{s}^{\varepsilon}(c, b, a)$, with

$$
\beta_{s}(c, b, a)=\frac{\omega(s, c, b, a) \cdot \omega(c, b, s, a)}{\omega(c, s, b, a) \cdot \omega(c, b, a, s)},
$$

and $\varepsilon=\operatorname{sgn}[\operatorname{det}(\vec{a}, \vec{b}, \vec{c}, \vec{s})]$. Using the 4-cocycle condition for $\omega$, it is straightforward to show that $\beta_{s}$ is a 3-cocycle. This shows that lifting all vertices of a tetrahedron produces a quantum phase which is only a 3-cocycle, for any given $\omega$.

Another quantity that appears naturally from a cubic geometry is $\gamma_{a, b}$, whose geometric meaning is shown in Fig. 6. It is defined from the 3 -cocycle $\beta_{a}$ as

$$
\gamma_{a, b}(c, d)=\frac{\beta_{a}(b, c, d) \beta_{a}(c, d, b)}{\beta_{a}(c, b, d)}
$$

It is straightforward to show that $\delta \gamma_{a, b}(c, d, e)=1$; namely, $\gamma_{a, b}$ is a 2-cocycle (see Appendix A). Further, from Eqs. (7) and (8), one can show that $\gamma_{a, b}(c, d)=\gamma_{b, a}^{-1}(c, d)$. This equality follows also from the geometry in Fig. 6.

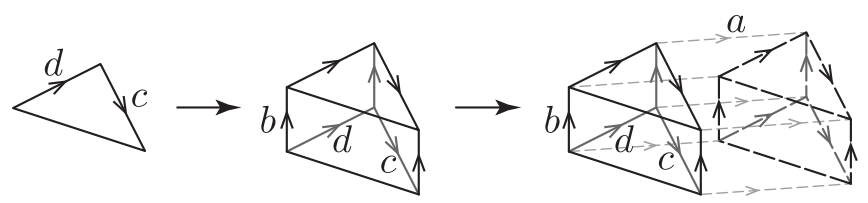

FIG. 6. Evolution from a triangle to a 4D manifold. Phase associated with this colored manifold is $\gamma_{a, b}^{\varepsilon}(c, d)$, where $\varepsilon=\operatorname{sgn}[\operatorname{det}(\vec{d}, \vec{c}, \vec{b}, \vec{a})]$. This phase can also be written as $\gamma_{b, a}^{\varepsilon^{\prime}}(c, d)$, where $\varepsilon^{\prime}=\operatorname{sgn}[\operatorname{det}(\vec{d}, \vec{c}, \vec{a}, \vec{b})]=-\varepsilon$. So, we conclude that $\gamma_{a, b}(c, d)=\gamma_{b, a}^{-1}(c, d)$. 


\section{GROUND STATE ON THREE-TORUS AND MEMBRANE OPERATORS}

\section{A. Exact models on three-torus}

We now put our model, Eq. (6), on the three-torus in $(3+1) \mathrm{D}$. It is important to note that the exactly solvable model has correlation length zero. Therefore, we can consider the simplest triangulation of a three-torus shown in Fig. 7. All eight cube vertices are identical due to periodic boundary conditions. It is triangulated by six tetrahedrons. There are three independent edges, which are assigned group elements $a, b, c \in G$, with $G$ a finite group. Edges with the same direction share the same group element value. The corresponding quantum state is labeled by $|a, b, c\rangle$. We also require $G$ to be Abelian for simplicity.

Since there is only one vertex, we denote the plaquette operator $\hat{B}_{p}$ simply as $\hat{B}$, which equals $(1 /|G|) \sum_{s \in G} \hat{B}^{s}$. The action of $\hat{B}^{s}$ on state $|a, b, c\rangle$ is

$$
\begin{aligned}
\hat{B}^{s}|a, b, c\rangle & =\frac{\gamma_{a, s}(b, c)}{\gamma_{a, s}(c, b)}|a, b, c\rangle . \\
& =\frac{\gamma_{a, b}(c, s)}{\gamma_{a, b}(s, c)}|a, b, c\rangle .
\end{aligned}
$$

We can directly write down the above result due to the observation that the $4 \mathrm{D}$ graph we obtain by acting with $\hat{B}^{s}$ is, in fact, made out of two copies of Fig. 6 [23]. Note that the $U(1)$ phase obtained by the action of $\hat{B}^{s}$ is a fully antisymmetric function of $a, b, c, s$, as can be seen both geometrically and algebraically.

\section{B. MES as ground-state basis}

First, we briefly review topological order in $(2+1)$ D. It is partially characterized by ground-state degeneracy on the torus [3]. One can understand this degeneracy by applying Wilson loop operators of distinct topological excitations winding around one of the noncontractible loops on the torus. From this point of view, one can see that the groundstate degeneracy equals the number of distinct topological superselection sectors.

Nonchiral topological order is fully determined by braiding statistics and topological spin of its topological

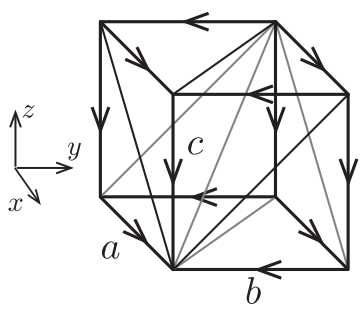

FIG. 7. The simplest triangulation of three-torus has a single vertex and three independent edges. Periodic boundary conditions are imposed on the cube. excitations [4]. Remarkably, one can read the information about excitations from ground state by using modular transformations $[2,13]$, namely, by considering the $\mathcal{S}, \mathcal{T}$ matrices of the $S, T$ transformation in the ground-state manifold. The dimension of $\mathcal{S}, \mathcal{T}$ equals the number of topological sectors. In a proper ground state basis, we can obtain the "canonical form" of $\mathcal{S}, \mathcal{T}$ matrices, for which the entries of the $\mathcal{S}$ matrix are the braiding statistics and the diagonal elements of $\mathcal{T}$ are the topological spins of quasiparticles. The ground-state basis for canonical $\mathcal{S}, \mathcal{T}$ matrices is formed by minimal entropy states [13].

We can extend these concepts to $(3+1) \mathrm{D}$. However, there is a major difference in this case: Topological excitations can be flux loops in $(3+1) \mathrm{D}$. Without loss of generality, we only consider the MES in the $z$ direction.

Inspired by the case of $(2+1) \mathrm{D}$ cohomological gauge theories discussed in Ref. [24], we find the MES in the $z$ direction as

$$
|a, b, \lambda\rangle=\frac{1}{\sqrt{|G|}} \sum_{c \in G} \tilde{\chi}_{\lambda}^{a, b}(c)|a, b, c\rangle,
$$

where $\tilde{\chi}_{\lambda}^{a, b}$ is a one-dimensional projective representation. Here, $\lambda$ labels different projective representations of the group $G$ (see Appendix A), and the 2-cocycle $\gamma$ from Eq. (8) plays the role of factor system of these projective representations:

$$
\tilde{\chi}_{\lambda}^{a, b}\left(c_{1}\right) \tilde{\chi}_{\lambda}^{a, b}\left(c_{2}\right)=\gamma_{a, b}\left(c_{1}, c_{2}\right) \tilde{\chi}_{\lambda}^{a, b}\left(c_{1} c_{2}\right) .
$$

We consider only the case of Abelian (one-dimensional) projective representations $\chi^{a, b}$ in this paper. This assumption implies that the 2-cocycle $\gamma_{a, b}$ is a 2-coboundary. We believe this is related to the physical assumption of Abelian statistics of loops. Note that our assumption about $\gamma_{a, b}$ implies that $\gamma_{a, b}(c, s)=\gamma_{a, b}(s, c)$, so that the phase factor in Eq. (9) is just identity, and states $|a, b, c\rangle$ for any $a, b$, $c \in G$ are in the ground-state manifold.

First, we verify that this state is indeed in the groundstate manifold. Acting with projection operator $\hat{B}$ on the state, we get

$$
\begin{aligned}
\hat{B}|a, b, \lambda\rangle & =\frac{1}{\sqrt{|G|^{3}}} \sum_{c \in G} \tilde{\chi}_{\lambda}^{a, b}(c) \sum_{s \in G} B^{s}|a, b, c\rangle \\
& =\frac{1}{\sqrt{|G|^{3}}} \sum_{c} \tilde{\chi}_{\lambda}^{a, b}(c) \cdot \frac{\gamma_{a, b}(c, s)}{\gamma_{a, b}(s, c)}|a, b, c\rangle \\
& =\frac{1}{\sqrt{|G|}} \sum_{c} \tilde{\chi}_{\lambda}^{a, b}(c)|a, b, c\rangle \\
& =|a, b, \lambda\rangle,
\end{aligned}
$$

where the second row uses Eq. (9), and in the third row, we use $\gamma_{a, b}(c, s)=\gamma_{a, b}(s, c)$, which follows from the above-mentioned assumptions. 


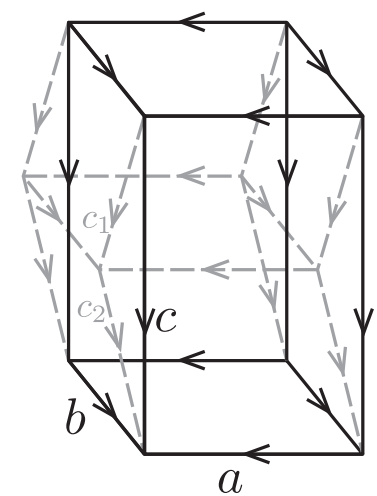

FIG. 8. Evolution from single vertex to two vertices.

Next, we prove that this state is indeed a MES in the $z$ direction. Let us retriangulate the three-torus, so that it has two unit cells in the $z$ direction. The ground state defined on this two unit-cell system can be evolved from that in one unit cell, as shown in Fig. 8:

$$
\begin{aligned}
|a, b, \lambda\rangle & =\frac{1}{\sqrt{|G|}} \sum_{c \in G} \tilde{\chi}_{\lambda}^{a, b}(c)|a, b, c\rangle \\
& =\frac{1}{\sqrt{|G|}} \sum_{c_{1}, c_{2} \in G} \tilde{\chi}_{\lambda}^{a, b}\left(c_{2} \cdot c_{1}\right) \gamma_{a, b}\left(c_{2}, c_{1}\right)\left|a, b, c_{1}, c_{2}\right\rangle \\
& =\frac{1}{\sqrt{|G|}} \sum_{c_{1}} \tilde{\chi}_{\lambda}^{a, b}\left(c_{1}\right) \sum_{c_{2}} \tilde{\chi}_{\lambda}^{a, b}\left(c_{2}\right)\left|a, b, c_{1}, c_{2}\right\rangle .
\end{aligned}
$$

As seen from the above, $|a, b, \lambda\rangle$ defined on two unit cells can be written as a direct product state. So, entanglement entropy of this state in the $z$ direction is zero, which must be minimum. We therefore conclude that this state is indeed a MES in the $z$ direction. Note that the entanglement entropy for this small system size vanishes due to a cancellation between the "area law" contribution and the topological contribution (see Ref. [13] and references therein), which will not occur for larger system sizes.

Similarly, it is easy to write down the MES in $x$ and $y$ directions:

$$
\begin{aligned}
& |\mu, b, c\rangle=\frac{1}{\sqrt{|G|}} \sum_{a \in G} \tilde{\chi}_{\mu}^{b, c}(a)|a, b, c\rangle, \\
& |a, \nu, c\rangle=\frac{1}{\sqrt{|G|}} \sum_{b \in G} \tilde{\chi}_{\nu}^{c, a}(b)|a, b, c\rangle,
\end{aligned}
$$

whose properties can be derived in the same way as above.

\section{Membrane operator}

Although we construct the MES in $(3+1) \mathrm{D}$, the physical picture is still unclear. Recall that in $(2+1) \mathrm{D}$ all MESs can be obtained from inserting ribbon operators (Wilson loop operators) into "trivial" MES, which corresponds to a topological trivial sector. In the following, we show that membrane operators are the relevant operators for such a procedure in $(3+1) \mathrm{D}$.

Let us start with the MES in the $z$ direction, $|a, b, \lambda\rangle$. Characteristically, in discrete gauge theory, we can interpret a group element as a label of flux loop. Consequently, a membrane, which is the $(3+1) \mathrm{D}$ analogue of the Dirac string, is also labeled by a group element. Further, a group representation labels a particle [10]. Then $|a, b, \lambda\rangle$ can be viewed as a state with membrane $a$ in the $y z$ plane and membrane $b$ in the $z x$ plane, as well as string $\lambda$ (worldline of particle) in the $z$ direction. So, it is natural to define a trivial MES as

$$
|e, e, \mathbb{1}\rangle=\frac{1}{\sqrt{|G|}} \sum_{c \in G}|e, e, c\rangle,
$$

where $e \in G$ is the identity element. Here, $\mathbb{1}$ means the trivial linear representation.

The central question becomes, What are the operators that send one MES to another? It is natural to assume that these operators correspond to membrane insertion in the $y z$ and $z x$ plane, as well as string insertion in the $z$ direction. Additionally, we expect that a string in the $x(y)$ direction can measure a membrane in the $y z(x z)$ plane while a membrane in the $x y$ plane will measure strings in the $z$ direction.

Following this intuition, we define membrane insertion operators in $y z, z x, x y$ planes, respectively, as shown in Fig. 9:

$$
\begin{aligned}
F_{b^{\prime}, c^{\prime}}^{u}|a, b, c\rangle & =\delta_{b b^{\prime}} \delta_{c c^{\prime}} \cdot \gamma_{b, c}^{-1}(u, a)|u a, b, c\rangle, \\
G_{c^{\prime}, a^{\prime}}^{v} & |a, b, c\rangle=\delta_{c c^{\prime}} \delta_{a a^{\prime}} \cdot \gamma_{c, a}^{-1}(v, b)|a, v b, c\rangle, \\
H_{a^{\prime}, b^{\prime}}^{w} & |a, b, c\rangle=\delta_{a a^{\prime}} \delta_{b b^{\prime}} \cdot \gamma_{a, b}^{-1}(w, c)|a, b, w c\rangle,
\end{aligned}
$$

where $u, v, w$ label the spatial planes of the membranes. Further, we can define

$$
\begin{aligned}
F_{u, \lambda}^{(z)} & =\sum_{b, c \in G} \tilde{\chi}_{\lambda}^{u, b}(c) F_{b, c}^{u}, \\
G_{v, \lambda}^{(z)} & =\sum_{c, a \in G} \tilde{\chi}_{\lambda}^{a, v}(c) G_{c, a}^{v},
\end{aligned}
$$
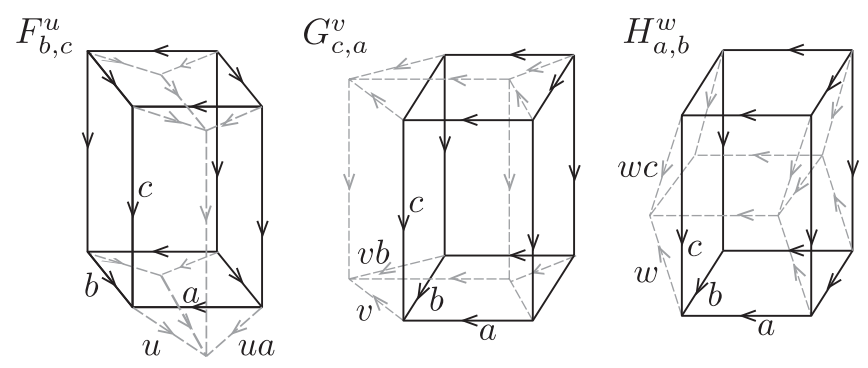

FIG. 9. The action of membrane operators. 
where we interpret $F_{u, \lambda}^{(z)}$ as inserting membrane $u$ (in the $y z$ plane) and string $\lambda$ in the $z$ direction, and we interpret $G_{v, \lambda}^{(z)}$ as inserting membrane $v$ (in the $z x$ plane) and string $\lambda$ in the $z$ direction. To confirm this, we act with these operators on state $|e, e, \mathbb{1}\rangle$, getting

$$
\begin{aligned}
& F_{u, \lambda}^{(z)}|e, e, \mathbb{1}\rangle=|u, e, \lambda\rangle, \\
& G_{v, \lambda}^{(z)}|e, e, \mathbb{1}\rangle=|e, v, \lambda\rangle .
\end{aligned}
$$

It is not hard to obtain the "fusion rule" of membranes and strings, namely,

$$
\begin{aligned}
& F_{u_{1}, \lambda_{1}}^{(z)} F_{u_{2}, \lambda_{2}}^{(z)}=F_{u_{1} u_{2}, \lambda_{3}}^{(z)}, \\
& G_{v_{1}, \lambda_{1}}^{(z)} G_{v_{2}, \lambda_{2}}^{(z)}=G_{v_{1} v_{2}, \lambda_{3}}^{(z)},
\end{aligned}
$$

and

$$
\begin{aligned}
& F_{u, \lambda_{1}}^{(z)} G_{v, \lambda_{2}}^{(z)}|a, b, \lambda\rangle=\left|u a, v b, \lambda_{3}\right\rangle, \\
& G_{v, \lambda_{1}}^{(z)} F_{u, \lambda_{2}}^{(z)}|a, b, \lambda\rangle=\left|u a, v b, \lambda_{3}\right\rangle,
\end{aligned}
$$

where the representation $\lambda_{3}$ is determined as the one in which every element $g \in G$ is represented by the product of numbers that represent $g$ in the $\lambda_{1}$ and $\lambda_{2}$ representations (note that the Abelian representations considered here are always one dimensional, i.e., just numbers). The fusion rules follow from the properties of the 2-cocycle $\gamma$, namely, assume $\tilde{\chi}_{\mu}^{a, b}$ is a projective representation with factor system $\gamma_{a, b}$,

$$
\tilde{\chi}_{\mu}^{a, b}\left(c_{1}\right) \cdot \tilde{\chi}_{\mu}^{a, b}\left(c_{2}\right)=\gamma_{a, b}\left(c_{1}, c_{2}\right) \cdot \tilde{\chi}_{\mu}^{a, b}\left(c_{1} \cdot c_{2}\right) .
$$

Then, it follows that

$$
\begin{aligned}
& \tilde{\chi}_{\mu_{1}}^{a, b_{1}}(c) \tilde{\chi}_{\mu_{2}}^{a, b_{2}} \gamma_{a, c}\left(b_{1}, b_{2}\right)=\tilde{\chi}_{\mu_{3}}^{a, b_{1} b_{2}}(c), \\
& \tilde{\chi}_{\mu_{1}}^{a_{1}, b}(c) \tilde{\chi}_{\mu_{2}, b}^{a_{2}, b} \gamma_{c, b}\left(a_{1}, a_{2}\right)=\tilde{\chi}_{\mu_{3}}^{a_{1} a_{2}, b}(c),
\end{aligned}
$$

where the representations $\mu_{1}, \mu_{2}, \mu_{3}$ are related in the same way as $\lambda_{1}, \lambda_{2}, \lambda_{3}$ just above.

Similarly to the above derivations, we can define

$$
\begin{aligned}
H_{w, \mu}^{(x)} & =\sum_{a, b} \tilde{\chi}_{\mu}^{b, w}(a) H_{a, b}^{w}, \\
H_{w, \mu}^{(y)} & =\sum_{a, b} \tilde{\chi}_{\nu}^{w, a}(b) H_{a, b}^{w},
\end{aligned}
$$

where $H^{(x)}\left(H^{(y)}\right)$ creates membrane in the $x y$ plane and string in the $x(y)$ direction. Acting with these operators on MES in the $z$ direction, we get

$$
\begin{aligned}
& H_{w, \mu}^{(x)}|a, b, \lambda\rangle=\frac{\tilde{\chi}_{\mu}^{b, w}(a)}{\tilde{\chi}_{\lambda}^{a, b}(w)}|a, b, \lambda\rangle, \\
& H_{w, \nu}^{(y)}|a, b, \lambda\rangle=\frac{\tilde{\chi}_{\nu}^{w, a}(b)}{\tilde{\chi}_{\lambda}^{a, b}(w)}|a, b, \lambda\rangle .
\end{aligned}
$$

It is then natural to interpret $H^{(x)}\left(H^{(y)}\right)$ as an operator that measures strings in the $z$ direction and membrane in the $y z(z x)$ plane.

We also write down the remaining two operators that send MES to MES for later convenience:

$$
\begin{aligned}
F_{u, \nu}^{(y)} & =\sum_{b, c} \tilde{\chi}_{\nu}^{c, u}(b) F_{b, c}^{u}, \\
G_{v, \mu}^{(x)} & =\sum_{c, a} \tilde{\chi}_{\mu}^{v, c}(a) G_{c, a}^{v} .
\end{aligned}
$$

\section{TOPOLOGICAL OBSERVABLES AND THEIR PHYSICAL INTERPRETATION}

\section{A. $\mathcal{S}$ and $\mathcal{T}$ matrices from modular transformations}

In this section, we calculate the Berry phase of ground states obtained during modular transformations. The derivation is largely a higher-dimensional generalization of the $(2+1)$ D case in Ref. [24].

In real space, we can write the modular transformations, Fig. 1(b), as

$$
\mathcal{S}=\left(\begin{array}{lll}
0 & 0 & 1 \\
1 & 0 & 0 \\
0 & 1 & 0
\end{array}\right), \quad \mathcal{T}^{31}=\left(\begin{array}{ccc}
1 & 0 & 0 \\
0 & 1 & 0 \\
1 & 0 & 1
\end{array}\right)
$$

The question is, What is the action of $\mathcal{S}$ and $\mathcal{T}$ on our exact models? We follow the strategy of Ref. [24], but generalize it to $(3+1) \mathrm{D}$. We consider a $T^{3} \times[0,1]$ manifold $\left(T^{3}\right.$ is three-torus), and put the initial ground state at $T^{3} \times 0$ and the final state at $T^{3} \times 1$. Then, we carefully triangulate the 4D manifold $T^{3} \times[0,1]$ and compute the quantum amplitude from the initial to the final state. After lengthy but straightforward calculations, we find

$$
\begin{aligned}
S|a, b, c\rangle & =|b, c, a\rangle, \\
T^{31}|a, b, c\rangle & =\beta_{b}^{-1}\left(a, a^{-1} c, a\right)\left|a, b, a^{-1} c\right\rangle .
\end{aligned}
$$

Now, we act by $T^{31}$ on MES in the $z$ direction:

$$
\begin{aligned}
T^{31}|a, b, \lambda\rangle & =\frac{1}{\sqrt{|G|}} \sum_{c} \tilde{\chi}_{\lambda}^{a, b}(c) \beta_{b}^{-1}\left(a, a^{-1}, a\right)\left|a, b, a^{-1} c\right\rangle \\
& =\tilde{\chi}_{\lambda}^{a, b}(a)|a, b, \lambda\rangle .
\end{aligned}
$$

We can see that $|a, b, \lambda\rangle$ is indeed an eigenstate of the $\mathcal{T}$ matrix. 
We can also get the $\mathcal{S}$-matrix element in the $z$ direction MES basis:

$$
\begin{aligned}
\left\langle a^{\prime}, b^{\prime}, \lambda^{\prime}|S| a, b, \lambda\right\rangle & =\frac{1}{|G|} \sum_{c, c^{\prime}} \frac{\tilde{\chi}_{\lambda}^{a, b}(c)}{\tilde{\chi}_{\lambda^{\prime}}^{a^{\prime}, b^{\prime}}\left(c^{\prime}\right)}\left\langle a^{\prime}, b^{\prime}, c^{\prime} \mid b, c, a\right\rangle \\
& =\frac{1}{|G|} \frac{\tilde{\chi}_{\lambda}^{a, b}\left(b^{\prime}\right)}{\tilde{\chi}_{\lambda^{\prime}}^{a^{\prime}, b^{\prime}}(a)} \cdot \delta_{a^{\prime} b} .
\end{aligned}
$$

Taking into account our assumption that $\gamma_{a, b}$ is a 2-coboundary, the projective representation $\tilde{\chi}$ can be rewritten as $\tilde{\chi}_{\mu}^{a b}(g)=\varepsilon_{a, b}(g) \cdot \chi_{\mu}(g)$, where $\chi_{\mu}(g)$ is an ordinary linear representation of $G$ and $\varepsilon_{a, b}$ is a 1-cocycle for which $\gamma_{a, b}=\delta \varepsilon_{a, b}$ (see Appendix A). Then, we get a factorized form:

$$
\left\langle a^{\prime}, b^{\prime}, \lambda^{\prime}|S| a, b, \lambda\right\rangle=\frac{1}{|G|} \frac{\chi_{\lambda}^{a, b}\left(b^{\prime}\right)}{\chi_{\lambda^{\prime}}^{a^{\prime}, b^{\prime}}(a)} \cdot \frac{\varepsilon_{a, b}\left(b^{\prime}\right)}{\varepsilon_{a^{\prime}, b^{\prime}}(a)} \cdot \delta_{a^{\prime} b} .
$$

While the physical meaning of this element is not so clear for the general case, it is instructive to see the simple case, where $a^{\prime}=b=e$. Then the 1-cocycle part of Eq. (31) is trivial and only $\chi_{\lambda}\left(b^{\prime}\right) / \chi_{\lambda^{\prime}}(a)$ is left. We can interpret this phase as an Aharonov-Bohm phase of particles going around a flux loop in three dimensions; namely, particle $\lambda$ sees flux loop $b^{\prime}$ and particle $\lambda^{\prime}$ sees flux loop $a$. In the following, we show how the most general form of the $\mathcal{S}$-matrix element, including the 1-cocycle contribution, can be interpreted as statistics of flux loops as well as particles.

\section{B. Braiding statistics from $\mathcal{S}$ matrix and triple linking number}

In $(2+1) \mathrm{D}$ spacetime dimensions, the $\mathcal{S}$-matrix elements can be directly related to quasiparticle braiding by considering quasiparticle tunneling operators $[3,13]$. One may ask if it is possible to capture the $\mathcal{S}$-matrix elements in $(3+1) \mathrm{D}$ spacetime dimensions using a loop braiding process by considering membrane operators. We show that this is indeed possible.

Let us review the $(2+1) \mathrm{D}$ case, mentioned in the Introduction. An $\mathcal{S}$-matrix element can be expressed as an overlap of two minimum entropy states, where every MES can be created by the action of a quasiparticle tunneling operator on the appropriate reference state [13]. Figure 2(a) depicts the MES overlap as a time sequence where application of a tunneling operator is represented as a spacetime event of a particle-antiparticle pair tunneling across the periodic system. We get two worldlines, since both MESs in the overlap contribute one. The occurrence of braiding can be revealed by realizing that the two worldlines in this process are linked when the $(2+1) \mathrm{D}$ spacetime process is embedded in three-dimensional space, as in Fig. 2(b). Therefore, an $\mathcal{S}$-matrix element describes braiding, which can be seen as linking of worldlines.
We now present an analogous interpretation of an $\mathcal{S}$-matrix element in $(3+1) \mathrm{D}$, using membrane operators and a process involving a triple linking of worldsheets in $(3+1) D$. Starting from the $\mathcal{S}$-matrix element in the MES basis in the $z$ direction, Eq. (30), it is straightforward to show that

$$
\begin{aligned}
\langle v, w, \mu|S| u, v, \lambda\rangle & =\langle\mu, v, w \mid u, v, \lambda\rangle \\
& =\frac{1 \tilde{\chi_{\lambda}^{u}, v}(w)}{|G| \tilde{\chi}_{\mu}^{v, w}(u)} \\
& =\left\langle 1, e, e\left|\left(G_{v, 1}^{(x)}\right)^{-1}\left(H_{w, \mu}^{(x)}\right)^{-1} F_{u, \lambda}^{(z)} G_{v, 1}^{(z)}\right| e, e, 1\right\rangle,
\end{aligned}
$$

where the last row follows from the definition of membrane operators. In contrast with the $(2+1) \mathrm{D}$ case, each MES is created by the action of two membrane operators on the reference state $|e, e, 1\rangle$. A membrane operator, such as Eqs. (18), (24), and (26), can be interpreted as a spacetime event of tunneling a flux-loop-antiflux-loop pair across a plane in the periodic system; for example, from Eq. (18), the $F_{u, \lambda}^{(z)}$ describes the tunneling of loop in the $z y$ plane. (A membrane also contains a string in its plane, which represents particle tunneling.)

The key question now becomes, What is a robust characterization of the process involving the four membrane operators in Eq. (32)? Inspired by the $(2+1) \mathrm{D}$ case, where embedding the process involving one-dimensional worldlines in three dimensions revealed their linking, we embed the process involving two-dimensional worldsheets into four dimensions. We find that three worldsheets have a nontrivial triple linking number, which is a topological invariant generalizing the linking number used for worldlines in $(2+1) \mathrm{D}$. The details of embedding the $(3+1) \mathrm{D}$ spacetime process into four-dimensional space are in Appendix B.

Here, we briefly summarize the TLN invariant.

The triple linking number $T l k_{M N P}$ of three oriented twodimensional surfaces $M, N, P$ smoothly embedded in four dimensions was defined in Ref. [16] as an analogue of the linking number of classical links. In our case, $M, N, P$ are the three flux-loop worldsheets in $(3+1) \mathrm{D}$.

$T l k_{M N P}$ is an integer topological invariant [25]. It can be nonzero only if the surfaces $M, N, P$ are distinct, and the Tlk obey the relations

$$
\begin{aligned}
T l k_{M N P}+T l k_{N P M}+T l k_{P M N} & =0, \\
T l k_{M N P}+T l k_{P N M} & =0,
\end{aligned}
$$

and are therefore fully determined by two integers [25].

There are different ways to calculate the TLN [25]. We describe the one that is most convenient for the braiding problem: One projects the surfaces $M, N, P$ from $(3+1) \mathrm{D}$ onto a three-dimensional slice using an arbitrary projection 

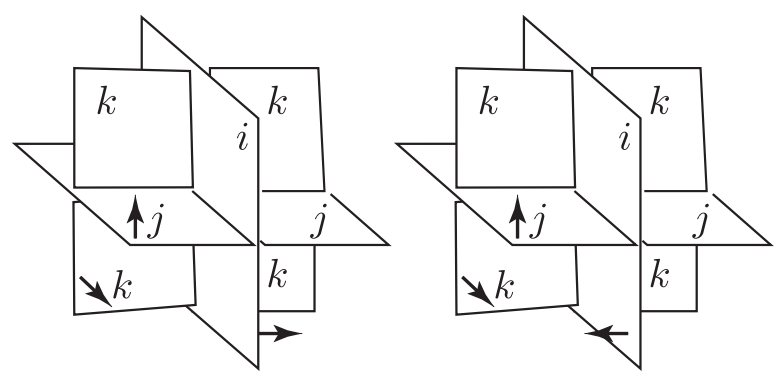

FIG. 10. A positive triple point (left) and a negative triple point (right), where we denote the orientations of sheets by their normals.

direction and looks for triple points, namely, points in the projected manifold where all three projected surfaces intersect. For each triple point $s$, one checks the stacking order of surfaces along the projection vector and assigns the top surface to $I_{s}$, the middle to $J_{s}$, and the bottom to $K_{s}$. Finally, the sign $\epsilon_{s}$ is calculated as the handedness of the three $I_{s}, J_{s}, K_{s}$ surface normals at the point $s$; see Fig. 10. Now, let $(I, J, K)$ be a permutation of the three surfaces $(M, N, P)$. Having the above information, $T l k_{I J K}$ equals the sum of $\epsilon_{s}$ over the points $s$ for which $I_{s}=I, J_{s}=J$, $K_{s}=K$. If no triple point contributes to a certain choice $I J K$, then $T l k_{I J K}=0$, and this has to be consistent with other values of $I^{\prime} J^{\prime} K^{\prime}$ according to Eq. (33).

The number of triple points and the stacking order of surfaces both depend on the chosen projection vector in $(3+1) \mathrm{D}$; however, the resulting TLN is topologically invariant.

Here, we present the TLN result, leaving the calculation details to Appendix B. We find that in the embedded process the worldsheets corresponding to membrane operators $F_{u, \lambda}^{(z)},\left(H_{w, \mu}^{(x)}\right)^{-1}$, and $\left(G_{v, 1}^{(x)}\right)^{-1}$ have triple linking, with

$$
\begin{aligned}
& T l k_{F H G}=T l k_{H F G}=1, \\
& T l k_{G H F}=T l k_{G F H}=-1, \\
& T l k_{F G H}=T l k_{H G F}=0 .
\end{aligned}
$$

We note that, even for general $(3+1) \mathrm{D}$ topological order, beyond cohomological models, the $\mathcal{S}$-matrix elements are given by MES overlaps, while the direct connection between MES overlap and triple linking of tunneling operator worldsheets, such as discussed above and demonstrated using Eq. (32), remains a general property based on the purely geometrical embedding construction. The only required ingredient is that the MESs of the topological order can be expressed using tunneling operators of looplike excitations, which we expect to be generally true in $(3+1) \mathrm{D}$.

\section{Braiding statistics from membrane operator algebra}

Returning to the $(2+1) \mathrm{D}$ case, as briefly reviewed in the Introduction, there is another way to relate an $\mathcal{S}$-matrix element to a topological property of the quasiparticle braiding process. Namely, the quasiparticle tunneling operators obey a nontrivial algebra, and a certain product of these operators gives the identity operator times a complex number, which equals an $\mathcal{S}$-matrix element $[3,12]$. Figure 2(c) depicts such a product of tunneling operators as a time sequence of events where particleantiparticle pairs tunnel across the periodic system, in the same fashion as in Fig. 2(a). The four worldlines in this process can be connected to reveal two linked worldlines, Fig. 2(d). Notice that this procedure can be done in the representation of the system as a parallelepiped with periodic boundary conditions, without need for embedding the $(2+1) \mathrm{D}$ spacetime in a three-dimensional space. One again confirms that the $\mathcal{S}$-matrix element describes braiding, which can in turn be seen as linking of worldlines.

Here, we present an analogous interpretation of a $(3+1) \mathrm{D} \mathcal{S}$-matrix element. The algebra of membrane operators follows from their definition:

$F_{u, \lambda}^{(z)} H_{w, \mu}^{(x)}|a, v, c\rangle=\frac{\tilde{\chi}_{\lambda}^{u, v}(w c) \tilde{\chi}_{\mu}^{v, w}(a)}{\gamma_{v, w c}(u, a) \gamma_{a, v}(w, c)}|u a, v, w c\rangle$,

$H_{w, \mu}^{(x)} F_{u, \lambda}^{(z)}|a, v, c\rangle=\frac{\tilde{\chi}_{\lambda}^{u, v}(c) \tilde{\chi}_{\mu}^{v, w}(u a)}{\gamma_{v, c}(u, a) \gamma_{u a, v}(w, c)}|u a, v, w c\rangle$.

Consider the membrane operator product $\left\langle G^{-1} F^{-1} H^{-1} F H G\right\rangle$, where the expectation value is obtained in state $|a, e, c\rangle$ with $a, c$ arbitrary [note that such a state is in the ground-state manifold, see after Eq. (11)]. Here, we label $F=F_{u, \lambda}^{(z)}, G=\sum_{c, a} G_{c, a}^{v}$, and $H=H_{w, \mu}^{(x)}$ for simplicity. Using Eq. (35), it is straightforward to show

$$
\left\langle G^{-1} F^{-1} H^{-1} F H G\right\rangle=\frac{\tilde{\chi}_{\lambda}^{u, v}(w)}{\tilde{\chi}_{\mu}^{v, w}(u)},
$$

so the quantum amplitude equals the $\mathcal{S}$-matrix element $\langle v, w, \mu|S| u, v, \lambda\rangle$ up to factor $|G|$.

As in the previous subsection, the membrane operators Eqs. (18), (24), and (26), are interpreted as events of tunneling a flux-loop-antiflux-loop pair across a plane in the periodic system; for example, from Eq. (18), the $F_{u, \lambda}^{(z)}$ describes the tunneling of loop in the $z y$ plane. The quantum amplitude in Eq. (36) can then be seen as the time sequence in Fig. 11.

Analogously to the $(2+1) \mathrm{D}$ case, where two worldlines, defined by a particle tunneling operator and its inverse, were connected into a single worldline, we can consider that the events defined by $F$ and $F^{-1}$ form a single worldsheet, and so on for $G$ and $H$. Having exactly three worldsheets, we calculate their TLN. Projecting the time sequence onto the three-dimensional space slice at time $t=-\infty$, we find eight triple intersection points of the projected worldsheets, Fig. 12. For simplicity of 


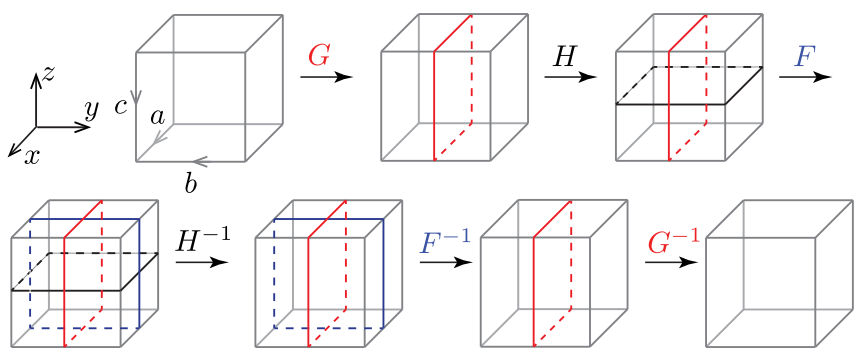

FIG. 11. Time sequence for process $\left\langle G^{-1} F^{-1} H^{-1} F H G\right\rangle$. The worldsheets in this process share the same topological properties as the three-flux-loop braiding process in Fig. 3.

presentation, we offset the spatial position of the inserted operator and its inverse; i.e., the membrane is moved slightly between the time of its appearance and disappearance. We check that this offset does not influence the result. Notice that the orientation of the membrane operator and its inverse is opposite; see Appendix B. A straightforward calculation from each triple point gives $a, c, e$ : $T l k_{F H G}=1, b: T l k_{H F G}=1, f: T l k_{G H F}=-1, e, g, h:$ $T l k_{G F H}=-1$, which is exactly the same triple linking number obtained from the $\mathcal{S}$-matrix calculation.

\section{Braiding process of three flux loops}

In previous subsections, we have interpreted the membrane operators as representing an instantaneous event of creating a loop-antiloop pair and expanding the loop across a plane in the periodic system until the loops annihilate. However, this kind of worldsheet evolution can be

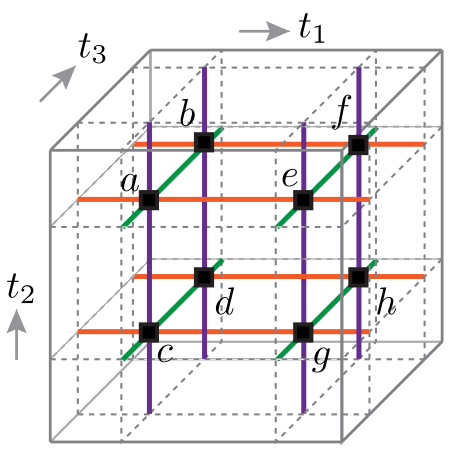

FIG. 12. Projection of the membrane process time sequence in Fig. 11 to three-dimensional space at $t=-\infty$. Lines show the pairwise intersections of projected worldsheets. Purple lines, $F$ and $G$ worldsheets; orange lines, $F$ and $H$; green lines, $G$ and $H$. Although there are eight triple points here, the triple linking is still the same as for the three-flux-loop braiding process, Fig. 13. The directions $t_{1,2,3}$ show the time ordering of contributions to projection from worldsheets $G, H, F$, and so clarify to which $T l k_{I J K}$ some triple point contributes [see after Eq. (33)]. For example, at point $a$, direction of $t_{1}, t_{2}, t_{3}$ shows that worldsheet projection at this point comes from $G$ rather than $G^{-1}, H^{-1}$ rather than $H, F^{-1}$ rather than $F$, respectively. Therefore, point $a$ contributes to $T l k_{F H G}$.

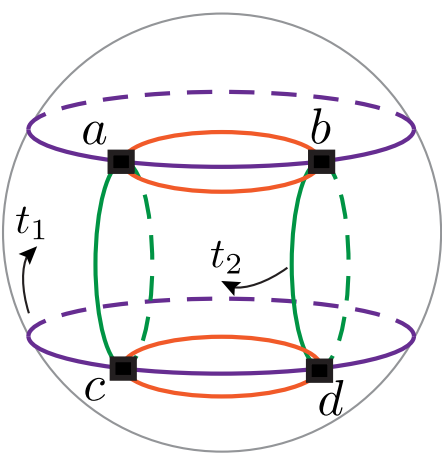

FIG. 13. Time sequence in Fig. 3 projected to threedimensional space at $t=-\infty$. Triple points are marked $a, b$, $c, d$. Lines show the pairwise intersections of projected worldsheets. Purple lines, $F$ and $G$ worldsheets; orange lines, $F$ and $H$; green lines, $G$ and $H$. The projected $G$ worldsheet in this figure takes the form of the sphere; $H$ and $F$ take the form of tori (not shown). The directions $t_{1,2}$ show the time ordering of contributions to projection from worldsheets $G, H$, and so clarify to which $T l k_{I J K}$ some triple point contributes [see after Eq. (33)]. For example, at point $c, t_{2}$ shows that projection of $H$ at this point comes at later times, so after $F$, while $t_{1}$ shows that $G$ comes from earlier times, so before both $F$ and $H$, altogether contributing to $T l k_{H F G}$. This process has the same triple linking number as the one in Fig. 12.

smoothly deformed to represent a more physically clear process. We, therefore, make a time sequence of the threeflux-loop braiding process that gives exactly the same nontrivial triple linking number as the membrane process, as shown in Fig. 3. By projecting this braiding time sequence, we get Fig. 13, in which it is straightforward to measure the TLN.

Triple point $a$ gives $\mathrm{Tlk}_{G H F}=-1$, triple point $b$ gives $\mathrm{Tlk}_{G F H}=-1$, triple point $c$ gives $\mathrm{Tlk}_{H F G}=1$, and triple point $d$ gives $\mathrm{Tlk}_{F H G}=1$. The obtained values of $T l k_{I J K}$ for the three-flux-loop braiding exactly match the membrane calculation result.

\section{EXAMPLES}

Here, we present the example of $G=Z_{2} \times Z_{2}$ cohomological gauge theories. Since $H^{4}(G, U(1))=Z_{2} \times Z_{2}$, they can represent different topological orders. This shows how the loop statistics can distinguish different topological orders.

It is convenient to label group $G$ elements $a$ as $\left(a_{1}, a_{2}\right)$, where $a_{i} \in\{0,1\}$. Group multiplication rule $a \cdot b$ is defined as $\left(\left\langle a_{1}+b_{1}\right\rangle,\left\langle a_{2}+b_{2}\right\rangle\right)$, where we introduce notation $\langle x\rangle \equiv x \bmod 2$.

Since the cohomology group is $H^{4}\left(Z_{2} \times Z_{2}, U(1)\right) \cong$ $Z_{2} \times Z_{2}$, it can be parametrized by 4-cocycles

$$
\left\{\omega_{i j} \mid i, j=0,1\right\}
$$

with multiplication rule 
$\omega_{i j}(a, b, c, d) \cdot \omega_{i^{\prime} j^{\prime}}(a, b, c, d)=\omega_{\left\langle i+i^{\prime}\right\rangle\left\langle j+j^{\prime}\right\rangle}(a, b, c, d)$.

The explicit form of these 4-cocycles is [26]

$\omega_{00}(a, b, c, d)=1$,

$\omega_{01}(a, b, c, d)=\exp \left[\frac{i \pi}{2} a_{1} b_{2}\left(c_{2}+d_{2}-\left\langle c_{2}+d_{2}\right\rangle\right)\right]$,

$\omega_{10}(a, b, c, d)=\exp \left[\frac{i \pi}{2} a_{2} b_{1}\left(c_{1}+d_{1}-\left\langle c_{1}+d_{1}\right\rangle\right)\right]$,

$\omega_{11}(a, b, c, d)=\omega_{01}(a, b, c, d) \cdot \omega_{10}(a, b, c, d)$.

It is straightforward to check that these $\omega$ indeed satisfy the 4-cocycle condition.

One can now work out the induced 3-cocycle $\beta_{a}$ and 2-cocycle $\gamma_{a, b}$ using their definitions in Eqs. (7) and (8). For the induced 3-cocycle, we get

$\beta_{00, a}(b, c, d)=1$,

$\beta_{01, a}(b, c, d)=\exp \left[\frac{i \pi}{2}\left(a_{1} b_{2}-a_{2} b_{1}\right)\left(c_{2}+d_{2}-\left\langle c_{2}+d_{2}\right\rangle\right)\right]$,

$\beta_{10, a}(b, c, d)=\exp \left[\frac{i \pi}{2}\left(a_{2} b_{1}-a_{1} b_{2}\right)\left(c_{1}+d_{1}-\left\langle c_{1}+d_{1}\right\rangle\right)\right]$,

$\beta_{11, a}(b, c, d)=\beta_{01, a}(b, c, d) \beta_{10, a}(b, c, d)$.

It follows that the 3-cocycle $\beta_{a}$ can be expressed as

$\beta_{a}(b, c, d)=\exp \left[\frac{i \pi}{2} P_{i j}^{a} b_{i}\left(c_{j}+d_{j}-\left\langle c_{j}+d_{j}\right\rangle\right)\right]$,

where $P_{i j}^{a}$ is some integer matrix. According to Ref. [27], then the induced 2-cocycle must be a coboundary $\gamma_{a, b}(c, d)=\delta \varepsilon_{a, b}(c, d)$, where

$$
\varepsilon_{a, b}(c)=\exp \left(\frac{i \pi}{2} P_{i j}^{a} b_{i} c_{j}\right) .
$$

Altogether, for inequivalent 4-cocycles, we get the induced 2-cocycle as

$$
\begin{aligned}
\varepsilon_{a, b}^{00}(c) & =1, \\
\varepsilon_{a, b}^{01}(c) & =\exp \left[\frac{i \pi}{2}\left(a_{1} b_{2} c_{2}-a_{2} b_{1} c_{2}\right)\right], \\
\varepsilon_{a, b}^{10}(c) & =\exp \left[\frac{i \pi}{2}\left(a_{2} b_{1} c_{1}-a_{1} b_{2} c_{1}\right)\right], \\
\varepsilon_{a, b}^{11}(c) & =\varepsilon_{a, b}^{01}(c) \cdot \varepsilon_{a, b}^{10}(c) .
\end{aligned}
$$

Now, we are ready to calculate statistics of loops and particles. We focus on

$$
\begin{aligned}
|G| \cdot\langle w, u, \nu|S| v, w, \mu\rangle & =\frac{\tilde{\chi}_{\mu}^{v, w}(u)}{\tilde{\chi}_{\nu}^{w, u}(v)} \\
& =\frac{\chi_{\mu}(u)}{\chi_{\nu}(v)} \cdot \frac{\varepsilon_{v, w}(u)}{\varepsilon_{w, u}(v)} .
\end{aligned}
$$

In the second equality, we defined

$$
\begin{aligned}
& \tilde{\chi}_{\mu}^{v w}(u)=\varepsilon_{v, w}(u) \cdot \chi_{\mu}(u), \\
& \tilde{\chi}_{\nu}^{w u}(v)=\varepsilon_{w, u}(v) \cdot \chi_{\nu}(v),
\end{aligned}
$$

where $\chi_{\mu}\left(\chi_{\nu}\right)$ is the one-dimensional linear representation of $Z_{2} \times Z_{2}$. One can easily check that the above definition of $\tilde{\chi}_{\mu}$ and $\tilde{\chi}_{\nu}$ is consistent, due to $\gamma_{a, b}$ being a 2-coboundary. Labeling $\mu=\left(\mu_{1}, \mu_{2}\right)$ as a $Z_{2} \times Z_{2}$ group element,

$$
\chi_{\mu}(u)=e^{i \pi\left(\mu_{1} u_{1}+\mu_{2} u_{2}\right)}=e^{i \pi \vec{\mu} \cdot \vec{u}} .
$$

First, let us consider the case $w=(0,0)$. In this case, only the $\chi_{\lambda}$ factors are nontrivial in the second line of Eq. (44), which is interpreted as a contribution from the Aharonov-Bohm phase of braiding particles around flux loops. In this case, the phase factor equals $e^{i \pi(\vec{\mu} \cdot \vec{u}-\vec{\nu} \cdot \vec{v})}$, which is independent of the choice of a cocycle. Namely, statistics between particles and loops cannot distinguish different phases.

Then, we turn to the general case. We get an additional phase factor $s_{l}$ beyond $e^{i \pi(\vec{\mu} \cdot \vec{u}-\vec{\nu} \cdot \vec{v})}$, and the $s_{l}$ factor comes from $\varepsilon$ in Eq. (44). In other words, it is present even when $\mu=\nu=0$, i.e., $\chi$ representations are trivial, so there are no charged particles. Therefore, $s_{l}$ represents statistics of flux loops. We list $s_{l}$ obtained from different 4-cocycles as follows:

(1) $\omega_{00}: s_{l}=1$,

(2) $\omega_{01}: s_{l}=e^{(i \pi / 2)\left[\left(u_{1} v_{2}+u_{2} v_{1}\right) w_{2}-2 u_{2} v_{2} w_{1}\right]}$,

(3) $\omega_{10}: s_{l}=e^{(i \pi / 2)\left[\left(u_{1} v_{2}+u_{2} v_{1}\right) w_{1}-2 u_{1} v_{1} w_{2}\right]}$,

(4) $\omega_{11}: s_{l}=e^{(i \pi / 2)\left[\left(u_{1} v_{2}+u_{2} v_{1}\right)\left(w_{1}+w_{2}\right)-2 u_{1} v_{1} w_{2}-2 u_{2} v_{2} w_{1}\right]}$.

We see that flux-loop braiding can indeed distinguish different topological orders in $(3+1) \mathrm{D}$, recalling here that the membrane operator expression is identified with a particular type of three-flux-loop braiding. In particular, according to Sec. IV, we can identify the flux loops (blue, red, black) in Fig. 3 with fluxes $(u, v, w)$ here, and there are no charges present.

Now, we turn to the $\mathcal{T}$-matrix element $\tilde{\chi}_{\lambda}^{u, v}(u)=$ $\left\langle u, v, \lambda\left|T^{31}\right| u, v, \lambda\right\rangle$. In the same way as above, we get

(1) $\omega_{00}: \tilde{\chi}_{\lambda}^{u, v}(u)=e^{i \pi \vec{\lambda} \cdot \vec{u}}$,

(2) $\omega_{01}: \tilde{\chi}_{\lambda}^{u, v}(u)=e^{i \pi \vec{\lambda} \cdot \vec{u}} e^{(i \pi / 2)\left(u_{1} v_{2} u_{2}-u_{2} v_{1} u_{2}\right)}$.

(3) $\omega_{10}: \tilde{\chi}_{\lambda}^{u, v}(u)=e^{i \pi \vec{\lambda} \cdot \vec{u}} e^{(i \pi / 2)\left(u_{2} v_{1} u_{1}-u_{1} v_{2} u_{1}\right)}$,

(4) $\omega_{11}: \tilde{\chi}_{\lambda}^{u, v}(u)=e^{i \pi \vec{\lambda} \cdot \vec{u}} e^{(i \pi / 2)\left(u_{1} v_{2}+u_{2} v_{1}\right)\left(u_{1}-u_{2}\right)}$.

While the $e^{i \pi \vec{\lambda} \cdot \vec{u}}$ can be interpreted as the Aharonov-Bohm phase of particles going around the loop, the remaining part also encodes information about loop statistics. While we do 
not have a proof at this time, we believe that this phase is related to the ribbon nature of flux loop, or in other words, to a thickness of the membrane.

\section{DISCUSSION AND CONCLUSIONS}

One of our main results is the construction of MES states on the three-torus for the $(3+1) \mathrm{D}$ cohomological gauge theory, which can be trivially generalized to an arbitrary number of unit cells. The $S, T$ transformation matrices take a simple form in this basis.

We discuss that the $\mathcal{S}$-matrix elements are directly related to the braiding of loop excitations. The $\mathcal{T}$-matrix elements, which are diagonal in the MES basis, correspond to the generalization of topological spin for loop excitations. Here, physically the loop excitations are generally expected to be ribbon excitations with two different loop edges. We expect that the geometrical interpretation of the $\mathcal{T}$-matrix elements is related to the braiding involving different loop edges.

Although we use exactly solvable models and $(3+1) \mathrm{D}$ topological quantum field theories to compute their $S, T$ matrices, these $(3+1) \mathrm{D} S, T$ matrices are, in principle, measurable quantities in practical model Hamiltonians. In particular, given a topologically ordered phase in $(3+1) \mathrm{D}$, with its topologically degenerate ground sector on threetorus $T^{3}$, one can first find a MES basis, similarly to the algorithms proposed in $(2+1) D$ [13]. For instance, for the $\mathcal{S}$-matrix element between two MESs $\left|\Xi_{i}\right\rangle$ and $\left|\Xi_{j}\right\rangle: S_{i j}$, one can perform the following thought numerical measurement. Because the topological properties do not depend on local geometry, we can assume that these ground states live on a cube with periodic boundary conditions. Then, one can consider the state rotated by $120^{\circ}$ along the (111) direction of the cube: $R_{120^{\circ}}\left|\Xi_{i}\right\rangle$. Because $R_{120^{\circ}}\left|\Xi_{i}\right\rangle$ and $\left|\Xi_{j}\right\rangle$ belong to the same topological phase, in the absence of symmetry, there should exist a Hamiltonian path $H(\tau)$ $(\tau \in[0,1])$ such that $\left|\Xi_{j}\right\rangle\left(\left|\Xi_{j}\right\rangle\right)$ are the ground state of $H(0)(H(1))$, and the ground-state sectors of $H(\tau)$ are adiabatically connected. One can then define a projection operator $\hat{P}_{\tau}$ into the ground-state sector of $H(\tau)$ for any given $\tau$. The many-body quantum amplitude related to the adiabatic time-evolution process of the $S$ transformation can be computed as $\left\langle\Xi_{j}\left|\hat{P}_{N-1 / N} \cdot \ldots \cdot \hat{P}_{2 / N} \cdot \hat{P}_{1 / N} R_{120^{\circ}}\right| \Xi_{i}\right\rangle$ as $N \rightarrow \infty$. This computation is a realization of the topological quantum field theory time evolution.

We expect that this quantum amplitude is related to the $\mathcal{S}$-matrix elements $s_{i j}$ at most by an overall ambiguity $U(1)$ phase $e^{i \theta}$, which is due to the nonuniversal local physics in the time evolution and a phase $e^{i \phi_{i}-i \phi_{j}}$, which is due to the gauge choice of $\left|\Xi_{i}\right\rangle,\left|\Xi_{j}\right\rangle$. Even with these ambiguities, such measurements can still be used to extract useful information about the $S, T$ matrices, which potentially could fully determine them.

Recently, there has been a lot of progress in relating topologically ordered phases to symmetry-protected topological and symmetry-enriched topological phases, for example, by partially or completely ungauging the gauge group $G$, i.e., by transformations between global and local symmetries [18,28-35]. We, therefore, expect that our work will be useful in characterization of symmetryprotected topological and symmetry-enriched topological phases too.

Our discussion is limited to the case in which both group $G$ and its projective representations are Abelian. These constraints are introduced here for simplicity rather than due to difficulty of principle. In the most general case, we expect $\tilde{\chi}$ to be the character of a projective representation, while modular transformations can easily be generalized, as shown in Ref. [24] for the $(2+1) \mathrm{D}$ case. In those cases, three-loop braiding may give a unitary matrix that corresponds to nonAbelian statistics of loops. In fact, we expect that the triple linking is relevant for any $(3+1) \mathrm{D}$ topological order, beyond cohomological models. Namely, as long as the MESs in a $(3+1) \mathrm{D}$ topologically ordered state can be written using tunneling operators of looplike excitations, which we believe should always be the case, our general geometrical approach based on spacetime embedding shows that MES overlaps, which appear as $\mathcal{S}$-matrix elements, will involve the triple linking of worldsheets defined by those tunneling operators.

Finally, we consider a trivial but ubiquitous example of $G=Z_{2}$. In this case, $H^{4}(G, U(1))=Z_{1}$, so the cocycle can be set to the identity map. The braiding phase $\tilde{\chi}_{\mu}^{v w}(u) / \tilde{\chi}_{\nu}^{w u}(v)$ reduces to a linear representation $\chi_{\mu}(u) / \chi_{\nu}(v)$, where group elements $u, v=0,1$ and $\mu, \nu=0,1$ label the representations of $Z_{2}$ :

$$
\chi_{\mu}(u)=e^{i \pi \mu u} .
$$

The braiding phase therefore equals $e^{i \pi(\mu u-\nu v)}$. There is no contribution from flux-loop braiding, since the 1-cocycle factors in Eq. (31) are trivial. In summary, the modular $S$ transformation for common $Z_{2}$ gauge theory in $(3+1) \mathrm{D}$ tells us that particles see a flux loop as a $\pi$ flux, and the flux loops themselves have trivial braiding.

Using the MES basis and Eqs. (29) and (30), we directly obtain the $\mathcal{S}$ and $\mathcal{T}$ matrices of $(3+1) \mathrm{D} Z_{2}$ theory in their canonical form:

$$
\begin{gathered}
\mathcal{S}=1 / 2\left(\begin{array}{cccccccc}
1 & 1 & 0 & 0 & 1 & 1 & 0 & 0 \\
1 & 1 & 0 & 0 & -1 & -1 & 0 & 0 \\
1 & -1 & 0 & 0 & 1 & -1 & 0 & 0 \\
1 & -1 & 0 & 0 & -1 & 1 & 0 & 0 \\
0 & 0 & 1 & 1 & 0 & 0 & 1 & 1 \\
0 & 0 & 1 & 1 & 0 & 0 & -1 & -1 \\
0 & 0 & 1 & -1 & 0 & 0 & 1 & -1 \\
0 & 0 & 1 & -1 & 0 & 0 & -1 & 1
\end{array}\right), \\
\mathcal{T}^{31}=\operatorname{Diag}(1,1,1,1,1,-1,1,-1),
\end{gathered}
$$


where the MES basis $|a, b, \lambda\rangle$, with $a, b, \lambda \in\{0,1\}$, is here naturally ordered according to binary numbers with digits $a b \lambda$. These matrices are consistent with the $\mathcal{S}$ and $\mathcal{T}$ matrices derived for the same theory in Ref. [8].

\section{ACKNOWLEDGMENTS}

This work was supported by the Alfred P. Sloan foundation and National Science Foundation under Grant No. DMR-1151440.

Note added.-Recently, we noticed a paper that also considers aspects of flux-loop braiding in $(3+1) \mathrm{D}$ [36].

\section{APPENDIX A: DEFINITION OF THE COHOMOLOGY GROUP AND PROJECTIVE REPRESENTATIONS}

We begin with a brief introduction to group cohomology. In this paper, we do not present the most general definition of group cohomology.

For a finite group $G$, and an Abelian group $M$ ( $M$ does not need to be finite or discrete), one can consider an arbitrary function that maps $n$ elements of $G$ to an element in $M ; \omega: G^{n} \rightarrow M$ or equivalently $\omega\left(g_{1}, g_{2}, \ldots, g_{n}\right) \in M$, $\forall g_{1}, g_{2}, \ldots g_{n} \in G$. Such a group function is called an $n$-cochain. The set of all $n$-cochains, which is denoted as $C^{n}(G, M)$, forms an Abelian group in the usual sense: $\left(\omega_{1} \cdot \omega_{2}\right)\left(g_{1}, g_{2}, \ldots, g_{n}\right)=\omega_{1}\left(g_{1}, g_{2}, \ldots, g_{n}\right) \cdot \omega_{2}\left(g_{1}, g_{2}, \ldots, g_{n}\right)$, in which the identity $n$-cochain is a group function whose value is always the identity in $M$.

One can define a mapping $\delta$ from $C^{n}(G, M)$ to $C^{n+1}(G, M): \forall \omega \in C^{n}(G, M)$, define $\delta \omega \in C^{n+1}(G, M)$ as

$$
\begin{aligned}
\delta \omega & \left(g_{1}, \ldots, g_{n+1}\right) \\
= & \omega\left(g_{2}, \ldots, g_{n+1}\right) \cdot \omega^{(-1)^{n+1}}\left(g_{1}, \ldots, g_{n}\right) \\
& \times \prod_{i=1}^{n} \omega^{(-1)^{i}}\left(g_{1}, \ldots, g_{i-1}, g_{i} \cdot g_{i+1}, g_{i+2}, \ldots, g_{n+1}\right) .
\end{aligned}
$$

It is easy to show that the mapping $\delta$ is nilpotent: $\delta^{2} \omega=1$ [here, 1 denotes the identity $(n+2)$-cochain]. In addition, for two $n$-cochains $\omega_{1}, \omega_{2}$, obviously, $\delta$ satisfies $\delta\left(\omega_{1} \cdot \omega_{2}\right)=\left(\delta \omega_{1}\right) \cdot\left(\delta \omega_{2}\right)$.

An $n$-cochain $\omega\left(g_{1}, \ldots g_{n}\right)$ is called an $n$-cocyle if and only if it satisfies the condition $\delta \omega=1$, where 1 is the identity element in $C^{n+1}(G, M)$. When this condition is satisfied, we also say that $\omega\left(g_{1}, \ldots g_{n}\right)$ is an $n$-cocycle of group $G$ with coefficients in $M$. The set of all $n$-cocycles, denoted by $Z^{n}(G, M)$, forms a subgroup of $C^{n}(G, M)$.

Not all different cocyles are inequivalent. Below, we define an equivalence relation in $Z^{n}(G, M)$. Because $\delta$ is nilpotent, for any $(n-1)$-cochain $c\left(g_{1}, \ldots, g_{n-1}\right)$, we can find the $n$-cocyle $\delta c$. And if an $n$-cocyle $b$ can be represented as $b=\delta c$, for some $c \in C^{n-1}(G, M), b$ is called an $n$-coboundary. The set of all $n$-coboundaries, denoted by
$B^{n}(G, M)$, forms a subgroup of $Z^{n}(G, M)$. Two $n$-cocycles $\omega_{1}, \omega_{2}$ are equivalent (denoted by $\omega_{1} \sim \omega_{2}$ ) if and only if they differ by an $n$-coboundary, $\omega_{1}=\omega_{2} \cdot b$, where $b \in B^{n}(G, M)$.

The $n$th cohomology group of group $G$, with coefficients in $M, H^{n}(G, M)$, is formed by the equivalence classes in $Z^{n}(B, M)$. More precisely, $H^{n}(G, M)=$ $Z^{n}(G, M) / B^{n}(G, M)$.

In this paper, we make a lot of use of 4-cocycles $\omega$. We always choose them to be in "canonical" form, which means that $\omega\left(g_{1}, g_{2}, g_{3}, g_{4}\right)=1$ if any of $g_{1}, g_{2}, g_{3}, g_{4}$ is equal to $\mathbb{1}$ (the identity element of group $G$ ). For any of the inequivalent cocycles mentioned above, it is always possible to choose a gauge such that $\omega$ becomes canonical [17].

In usual unitary group representations, each group element $g$ in $G$ is represented by a unitary matrix $D(g)$, which satisfies $D\left(g_{1}\right) \cdot D\left(g_{2}\right)=D\left(g_{1} \cdot g_{2}\right)$. The projective representations of the group $G$ are defined by modifying this relation by a phase factor $\omega\left(g_{1}, g_{2}\right) \in U(1)$ :

$$
D\left(g_{1}\right) \cdot D\left(g_{2}\right)=\omega\left(g_{1}, g_{2}\right) D\left(g_{1} \cdot g_{2}\right),
$$

where $\omega\left(g_{1}, g_{2}\right)$ is a function of $g_{1}, g_{2}$, called a factor system [37]. A factor system cannot be arbitrary. In order to satisfy the associativity condition $\left[D\left(g_{1}\right) \cdot D\left(g_{2}\right)\right] \cdot D\left(g_{3}\right)=$ $D\left(g_{1}\right) \cdot\left[D\left(g_{2}\right) \cdot D\left(g_{3}\right)\right]$, the factor system must satisfy the equation

$\omega\left(g_{1}, g_{2}\right) \cdot \omega\left(g_{1} \cdot g_{2}, g_{3}\right)=\omega\left(g_{2}, g_{3}\right) \cdot \omega\left(g_{1}, g_{2} \cdot g_{3}\right)$.

This relation is precisely the condition for $\omega$ to be a 2-cocycle [the condition is $\delta \omega=1$ in Eq. (A1) for $n=2$ ]. If $\omega\left(g_{1}, g_{2}\right)$ is a 2-coboundary, it can be written as $\omega\left(g_{1}, g_{2}\right)=c\left(g_{1}\right) \cdot c\left(g_{2}\right) / c\left(g_{1} \cdot g_{2}\right)$ for a certain 1-cochain $c(g)$. If two 2 -cocyles, $\omega_{1}, \omega_{2}$, differ by a 2 -coboundary,

$$
\omega_{1}\left(g_{1}, g_{2}\right)=\omega_{2}\left(g_{1}, g_{2}\right) \cdot \frac{c\left(g_{1}\right) \cdot c\left(g_{2}\right)}{c\left(g_{1} \cdot g_{2}\right)},
$$

it is obvious that they correspond to equivalent projective representations, because one can absorb the 1-cochain into $D(g)$ by redefining $\tilde{D}(g)=c(g) \cdot D(g)$, after which the two factor systems become the same (this is actually the definition of equivalent projective representations). Therefore, the $H^{2}(G, U(1))$ also classifies all inequivalent (factor systems of) projective representations.

\section{APPENDIX B: GEOMETRICAL INTERPRETATION OF $\mathcal{S}$ MATRIX AND TRIPLE LINKING CALCULATION CONVENTIONS}

In this section, we present the details of embedding the $(3+1) \mathrm{D}$ spacetime in a four-dimensional Euclidean space $\mathbb{R}^{4}$, which is applied to the third line of Eq. (32) to show that in the embedded space worldsheets exhibit triple linking. The embedding is a generalization of the $(2+1) \mathrm{D}$ 
case shown in Figs. 2(a) and 2(b). The space manifold is a three-torus $T^{3}$, topologically a product of three circles $S_{(1)}^{1} \times S_{(2)}^{1} \times S_{(3)}^{1}$, with $1,2,3$ the spatial directions. At a given moment of time, this space is embedded in $\mathbb{R}^{4}$ to form the manifold $\Sigma$, which is simply the surface of a three-torus:

$$
\begin{aligned}
X & =\left[r_{1}+\left(r_{2}+r_{3} \cos \gamma\right) \cos \beta\right] \cos \alpha, \\
Y & =\left[r_{1}+\left(r_{2}+r_{3} \cos \gamma\right) \cos \beta\right] \sin \alpha, \\
Z & =\left(r_{2}+r_{3} \cos \gamma\right) \sin \beta, \\
W & =r_{3} \sin \gamma,
\end{aligned}
$$

where $r_{i}$ is radius for $S_{(i)}^{1}$, which is constant at fixed time, and $(X, Y, Z, W)$ are Cartesian coordinates in $\mathbb{R}^{4}$. The $\alpha, \beta$, $\gamma$ are angles of $S_{(1)}^{1}, S_{(2)}^{1}, S_{(3)}^{1}$, respectively, and correspond to Cartesian spatial coordinates in a cube with periodic boundary conditions. Without loss of generality, for every moment in time $t$, we set $r_{1}>r_{2}>r_{3}$. The embedding of different time slices is chosen such that the manifold $\Sigma$ at later times always contains all $\Sigma$ from the past. More precisely, $\left[\Sigma \times\left(-\infty, t_{1}\right)\right] \subset\left[\Sigma \times\left(-\infty, t_{2}\right)\right]$, for any $t_{1}<t_{2}$. Finally, the four-dimensional manifold $M=\Sigma \times(-\infty, \infty)$ covers the whole $\mathbb{R}^{4}$ space.

As $t \rightarrow-\infty, r_{2}, r_{3} \rightarrow 0$, so the space manifold shrinks to a circle in the $X Y$ plane: $X^{2}+Y^{2}=r_{1}[t=-\infty]^{2}$. As $t \rightarrow \infty, r_{1}, r_{2}, r_{3} \rightarrow \infty$, and the space manifold $\Sigma$ asymptotically approaches the $W$ axis, which can be viewed as the $S_{(3)}^{1}$ circle $\left(\gamma\right.$ circle) with $r_{3}=\infty$. Using all time $(-\infty, \infty)$ with these asymptotic limits in the embedding is useful for consistently removing ambiguities in the embedding of events corresponding to membrane operators, as will soon become clear.

After embedding the spacetime manifold itself, we proceed to an $\mathcal{S}$-matrix element as a sequence of spacetime events. For convenience, we repeat Eq. (32) here:

$$
\begin{aligned}
\langle v, w, \mu|S| u, v, \lambda\rangle & =\langle\mu, v, w \mid u, v, \lambda\rangle \\
& =\left\langle 1, e, e\left|\left(G_{v, 1}^{(x)}\right)^{-1}\left(H_{w, \mu}^{(x)}\right)^{-1} F_{u, \lambda}^{(z)} G_{v, 1}^{(z)}\right| e, e, 1\right\rangle .
\end{aligned}
$$

It is useful to think about this equation as a two-step process in time: from $t=-\infty$ to $t=0$ and from $t=0$ to $t=\infty$. At $t=0^{-}$, the system is in state $|u, v, \lambda\rangle$, obtained by insertion of membranes $G_{v, 1}^{(z)}$ and $F_{u, \lambda}^{(z)}$ at some time $t<0$ into the trivial MES $|e, e, 1\rangle$. On the other hand, the interval from $t=0$ to $t=\infty$ can be interpreted as the conjugate of an appropriate history from $t=-\infty$ to $t=0$. This gives us the bra $\langle\mu, v, w|$ by simply inserting membranes $G$ and $H$ into the trivial MES at $t=\infty$. Joining the two histories at $t=0$, the product of bra and ket is obtained. So the quantum amplitude that equals the $\mathcal{S}$-matrix element is naturally interpreted as a sequence of spacetime events.

Crucially, the coordinates $x, y, z$ used for the membranes are in the original three-dimensional space, and we yet have to consistently identify them with the $\alpha, \beta, \gamma$ coordinates that were defined above in the embedding of space into $\mathbb{R}^{4}$. It turns out that $F$ creates a sheet covering $S_{(1)}^{1} \times S_{(2)}^{1}$, $G$ creates the sheet $S_{(3)}^{1} \times S_{(1)}^{1}$, and membrane $H$ covers $S_{(2)}^{1} \times S_{(3)}^{1}$, as we show below. In other words, if we cut open the three-torus $T^{3}$ to a cube, then the $x$ axis corresponds to $\gamma$, the $y$ axis corresponds to $\beta$, and the $z$ axis corresponds to $\alpha$, remembering that $F$ is membrane in the $y z$ plane, $G$ is membrane in the $z x$ plane, and $H$ is membrane in the $x y$ plane.

To see this, we start from the trivial MES ket $|e, e, 1\rangle=\frac{1}{\sqrt{|G|}} \sum_{c}|e, e, c\rangle$, where the three labels inside the ket correspond to the three directions $x, y, z$. Consider the corresponding limit $t \rightarrow-\infty$, in which the $\beta, \gamma$ circles shrink to points, while the $\alpha$ circle remains at finite radius $r_{1}$. Therefore, the product of the group elements along the $\alpha$ circle remains unconstrained in this limit. On the other hand, we conclude that the product of the group elements along the $\beta$ or $\gamma$ direction has to be identity $e$ to be shrinkable to a point. More concretely, consider a consistent triangulation of entire $\mathbb{R}^{4}$, so that the nested $\Sigma$ manifolds coming from different times are connected by the consistent triangulation. Then, the $\beta$ and $\gamma$ circles at times $t<0$ are bounding consistently triangulated disks, and as they shrink, the zero-flux rule through the disks would force the product of group elements along the circles to identity $e$. Altogether, only the $\alpha$ coordinate can be identified as $z$, while $\beta, \gamma$ correspond to $x, y$ (not necessarily in that order).

We can perform a similar analysis for bra $\langle 1, e, e|$ and the corresponding limit $t \rightarrow \infty$. The finite loop at $t=\infty$ is $S_{(3)}^{1}$, the $\gamma$ circle. Then, it is easy to confirm that $x$ corresponds to $\gamma$. So, the remaining axis $y$ corresponds to $S_{(2)}^{1}$, i.e., to the $\beta$ circle.

Finally, we discuss some conventions we have chosen in the calculations of TLN in Secs. I and IV C. As described after Eq. (33), the TLN requires the calculation of normal vectors to worldsheets, which are projected from four to three dimensions. The overall sign of $T l k_{I J K}$ therefore depends on a precise definition of orientations of worldsheets, but these orientations are not inherent in the membrane operators and we need to choose them consistently. The $F$ membrane operator defines a sheet in the $y z$ plane, and we assign it the ordered pair $(z, y)$. After a projection to three dimensions, the derivatives with respect to $z, y$ define the sheet tangent vectors $\vec{n}_{1}, \vec{n}_{2}$, respectively, and the normal vector to sheet $F$ is chosen as $\vec{n}_{1} \times \vec{n}_{2}$. For $F^{-1}$, the normal is defined with opposite sign. In Sec. IV C, the total worldsheet composed of $F$ and $F^{-1}$ therefore has a 
consistent normal vector throughout. In the exact same fashion, we assign to the $G$ sheet the pair $(x, z)$, and to $H$ the $(y, x)$ pair. Finally, we note that the Jacobian of the transformation in Eq. (B1) has a negative determinant, so the embedding reverses the handedness of the coordinates in the three-dimensional projected space. Because of this, the TLN values calculated from the embedding are additionally multiplied by -1 .

[1] X.-G. Wen, Topological Orders and Edge Excitations in Fractional Quantum Hall States, Adv. Phys. 44, 405 (1995).

[2] X. G. Wen, Topological Orders in Rigid States, Int. J. Mod. Phys. B 04, 239 (1990).

[3] X. G. Wen and Q. Niu, Ground-State Degeneracy of the Fractional Quantum Hall States in the Presence of a Random Potential and on High-Genus Riemann Surfaces, Phys. Rev. B 41, 9377 (1990).

[4] X. G. Wen and A. Zee, Classification of Abelian Quantum Hall States and Matrix Formulation of Topological Fluids, Phys. Rev. B, 46, 2290 (1992).

[5] F. A. Bais and J. C. Romers, The Modular S-Matrix as Order Parameter for Topological Phase Transitions, New J. Phys. 14, 035024 (2012).

[6] More precisely, $S L(2, Z)$ is the mapping class group of the two-torus, i.e., the group of isotopy classes of automorphisms of the torus. The mapping class group is formed by Dehn twists of the torus.

[7] S. M. Trott, A Pair of Generators for the Unimodular Group, Can. Math. Bull. 5, 245 (1962).

[8] H. Moradi and X.-G. Wen, Universal Wave Function Overlap and Universal Topological Data from Generic Gapped Ground sSates, arXiv:1401.0518v1.

[9] R. Dijkgraaf and E. Witten, Topological Gauge Theories and Group Cohomology, Commun. Math. Phys. 129, 393 (1990).

[10] F. Alexander Bais, P. van Driel, and M. de Wild Propitius, Anyons in Discrete Gauge Theories with Chern-Simons Terms, Nucl. Phys. B393, 547 (1993).

[11] Discrete gauge theories can only describe nonchiral states having quasiparticles with integer quantum dimension. Also, some distinct phases can differ by a physically irrelevant relabeling of quasiparticles.

[12] M. Oshikawa and T. Senthil, Fractionalization, Topological Order, and Quasiparticle Statistics, Phys. Rev. Lett. 96, 060601 (2006).

[13] Y. Zhang, T. Grover, A. Turner, M. Oshikawa, and A. Vishwanath, Quasiparticle Statistics and Braiding from Ground-State Entanglement, Phys. Rev. B 85, 235151 (2012).

[14] J. C. Baez, D. K. Wise, and A. S. Crans, Exotic Statistics for Strings in 4d BF Theory, Adv. Theor. Math. Phys. 11, 707 (2007).

[15] A. Niemi, Exotic Statistics of Leapfrogging Vortex Rings, Phys. Rev. Lett. 94, 124502 (2005).

[16] J. Scott Carter, D. Jelsovsky, S. Kamada, and M. Saito, Quandle Homology Groups, Their Betti Numbers, and Virtual Knots, J. Pure Appl. Algebra 157, 135 (2001).
[17] X. Chen, Z.-C. Gu, Z.-X. Liu, and X.-G. Wen, Symmetry Protected Topological Orders and the Group Cohomology of Their Symmetry Group, Phys. Rev. B 87, 155114 (2013).

[18] A. Mesaros and Y. Ran, Classification of Symmetry Enriched Topological Phases with Exactly Solvable Models, Phys. Rev. B 87, 155115 (2013).

[19] M. Nakahara, Geometry, Topology, and Physics (Institute of Physics, Bristol, England, 2003).

[20] H. S. M. Coxeter, Introduction to Geometry (John Wiley and Sons, New York, 1989).

[21] The 4D coordinate system $(x, y, z, w)$ itself has a chirality, analogously to the handedness of a 3D coordinate system, and if it changes, the $\varepsilon$ also changes sign.

[22] M. Wakui, On Dijkgraaf-Witten Invariant for 3-Manifolds, Osaka J. Math. 29, 675 (1992).

[23] The precise way in which the $\hat{B}^{s}$ operator acts in the threetorus with a single vertex (Fig. 7) is obtained by first adding the (identical) edge rising into the fourth dimension from all eight (identical) corners of the cube. Then, for each of six tetrahedra forming the cube, one obtains the object in Fig. 5. This object can be triangulated into four 4-simplices, giving a total of 24 4-simplices, which contribute to the quantum amplitude of $\hat{B}^{s}$. An insightful shortcut is based on Fig. 6, which gives in elegant form the total amplitude contribution coming from half of the cube.

[24] Y. Hu, Y. Wan, and Y.-S. Wu, Twisted Quantum Double Model of Topological Phases in Two-Dimension, arXiv:1211.3695v1.

[25] S. Carter, S. Kamada, and M. Saito, Surfaces in 4-Space (Springer-Verlag, Berlin, 2004).

[26] X. Chen, Y.-M. Lu, and A. Vishwanath, Symmetry Protected Topological Phases from Decorated Domain Walls, Nat. Commun. 5, 3507 (2014).

[27] M. de Wild Propitius, Topological Interactions in Broken Gauge Theories, arXiv:hep-th/9511195v1.

[28] M. Levin and Z.-C. Gu, Braiding Statistics Approach to Symmetry-Protected Topological Phases, Phys. Rev. B 86, 115109 (2012).

[29] X. Chen, F. J. Burnell, A. Vishwanath, and L. Fidkowski, Anomalous Symmetry Fractionalization and Surface Topological Order, arXiv:1403.6491v1.

[30] Y. Gu, L.-Y. Hung, and Y. Wan, A Unified Framework of Topological Phases with Symmetry, arXiv:1402.3356v2.

[31] P. Ye and J. Wang, Symmetry-Protected Topological Phases with Charge and Spin Symmetries: Response Theory and Dynamical Gauge Theory in Two and Three Dimensions, Phys. Rev. B, 88, 235109 (2013).

[32] X. Chen and A. Vishwanath, "Gauging” Time Reversal Symmetry in Tensor Network States, arXiv:1401.3736v1.

[33] L.-Y. Hung and Y. Wan, String-Net Models with $Z_{N}$ Fusion Algebra, Phys. Rev. B 86, 235132 (2012).

[34] M. P. Zaletel, Detecting Two Dimensional Symmetry Protected Topological Order in a Ground State Wave Function, arXiv:1309.7387v2.

[35] C. Xu, Three-Dimensional $Z_{2}$ Topological Phases Enriched by Time-Reversal Symmetry, Phys. Rev. B 88, 205137 (2013).

[36] C. Wang and M. Levin, Braiding Statistics of Loop Excitations in Three Dimensions, arXiv:1403.7437v1.

[37] I. Martin Isaacs, Character Theory of Finite Groups (Academic Press, New York, 1976). 